\title{
LINEAR BUCKLING ANALYSIS OF PERFORATED COLD-FORMED STEEL STORAGE RACK COLUMNS BY MEANS OF THE GENERALISED BEAM THEORY
}

\author{
M. Casafont*, J. Bonada*, M.M. Pastor*, F. Roure*, A. Susín** \\ * Department of Strength of materials and Structural Engineering, Escola Tècnica Superior d'Enginyeria \\ Industrial de Barcelona, Universitat Politècnica de Catalunya (UPC). \\ ** Department of Applied Mathematics, Escola Tècnica Superior d’Enginyeria Industrial de Barcelona, \\ Universitat Politècnica de Catalunya (UPC).
}

Keywords: Generalised Beam Theory (GBT), thin-walled members, steel-storage rack columns, perforations.

\begin{abstract}
The investigation attempts to adapt a beam finite element procedure based on the Generalised Beam Theory (GBT) to the analysis of perforated columns. The presence of perforations is taken into account through the use of two beam elements with different properties, for the nonperforated and perforated parts of the member. Each part is meshed with its corresponding finite element and, afterwards, they are linked by means of constraint equations. Linear buckling analyses on steel storage rack columns are carried out to demonstrate how the proposed procedure should be applied. Some practical issues are discussed, such as the GBT deformation modes to be included in the analyses, or the optimum finite element discretization. The resulting buckling loads are validated by comparison with the values obtained in analyses performed using shell finite element models. Finally, it is verified that the buckling loads produced with the proposed method are rather accurate.
\end{abstract}

\section{INTRODUCTION}

The article presents a numerical procedure to carry out linear buckling analysis of coldformed steel members with multiple perforations evenly distributed along their length. The procedure has been derived to calculate the buckling loads of the columns used in steel storage rack structures. These calculations are performed by means of a beam finite element procedure developed on the basis of the Generalised Beam Theory (GBT).

Nowadays, three numerical methods are commonly applied to carry out linear buckling analyses of thin-walled members: the Finite Element Method (FEM), the Finite Strip Method (FSM), and the Generalised Beam Theory. The FEM is the most versatile, since it can be easily adapted to complex geometries and different load and member end conditions. However, its computational cost is high, and it is usually implemented in software that is difficult to learn and use. On the contrary, FSM and GBT analyses can be carried out with more accessible and easy to use programs, such as CUFSM ${ }^{1}$ and GBTUL $^{2}$. The latest advances in these programs have allowed FSM and GBT to become a very good alternative to FEM. Nowadays, they offer an acceptable adaptability to different analysis conditions at low computational cost ${ }^{3,4}$.

The problem when both GBT and FSM are applied to rack columns is that they cannot deal with perforations. These methods can only consider variations at the cross-section level, and cannot easily reproduce any discrete variation along the length of the member. However, in view of the advantages that the use of GBT and FSM involve, it is worth trying to expand 
their application to perforated rack columns. This is the aim of the investigation presented in the article.

The reduced thickness approach has been recently applied by different authors to tackle the analysis of members with rack type perforations using the $\mathrm{FSM}^{5-7}$. It consists in reducing the thickness of some parts of the cross-section to take into account the effect of holes. The reduced thickness used in the perforated parts is provided by equations that have been previously calibrated in different investigations ${ }^{5}$. This approach is very practical, since it can be applied using the existing FSM software. The only difference with respect to the analysis of an unperforated member lies in the fact that the cross-section model has to include some segments with thinner thickness. The main disadvantage of the method is that it might be inaccurate when applied to members with cross-sections and holes different from those used in the calibration of the reduced thickness equations.

A more general method is proposed in Eccher et al. ${ }^{8}$, where the formulation of the FSM analysis procedure is modified to include directly the holes in the model. This can be done through the application of the isoparametric spline finite strip method, which results in excellent elastic buckling load estimations for rack columns with different types of holes (rectangular, elliptical and diamond shaped holes).

In the present investigation, holes are also taken into account at the derivation stage of the analysis procedure. Therefore, a general method is also proposed, but it is developed in the frame of the Generalised Beam Theory, with which the authors have already carried out some investigations in the past ${ }^{9,10}$.

The article is devoted to show how the standard GBT beam finite element method has been adapted to consider perforations. The proposed approach, presented in Section 2, consists in meshing the perforated and non-perforated parts of the member with two different finite elements, the perforated and non-perforated finite element, respectively. The GBT mechanical properties of the perforated element have been reduced to take into account the holes, but no calibration is needed as shown in Section 2.1. Afterwards, the perforated and non-perforated parts are linked by means of constraint equations on the degrees of freedom of the finite elements. In this sense, the proposed procedure is similar to the one recently presented by Cai and Moen ${ }^{11}$. Some analysis examples are included in Section 3 to show the key points and the performance of the new method. Finally, the conclusions of Section 4 will close the paper. It should be noted that the investigation has only been focused on linear buckling analysis of compressed members.

\section{THEORETICAL BASIS FOR THE ANALYSIS OF PERFORATED MEMBERS VIA GBT}

Generally speaking, the method presented in this article follows the standard GBT procedure when deriving the cross-section mechanical properties and creating the finite element stiffness matrices. The presence of holes only involves small changes into these two GBT operations, as it is shown in Sections 2.1 and 2.2. The proposed approach differs from the usual GBT procedure in the process of assembly of the element stiffness matrices to obtain the global stiffness matrix. This particular matrix assembly is described in Section 2.3.

\subsection{Cross-section analysis}

The first step in any GBT calculation is the cross-section analysis, from which the deformation modes and the modal GBT cross-section properties are determined ${ }^{12,13}$. The deformation modes are usually grouped into four different classes ${ }^{14}$ : (i) conventional modes, including global modes (G), distortional modes (D) and local modes (L); (ii) natural shear modes (NS); (iii) transverse-extension modes (TE); and (iv) local shear modes (LS). Each 
mode is defined by means of a set of three functions $u(s), v(s)$ and $w(s)$, corresponding to the mid-plane wall displacements of the cross-section in the local $x, s$ and $z$ coordinate system, respectively (Fig. 1). For example, some of the GBT modes of a channel cross-section are illustrated in Fig. 2.

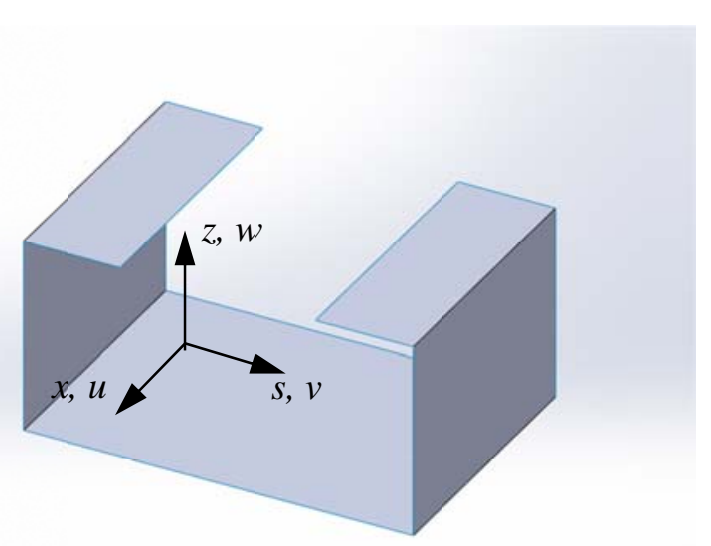

Fig. 1. GBT local coordinate system.

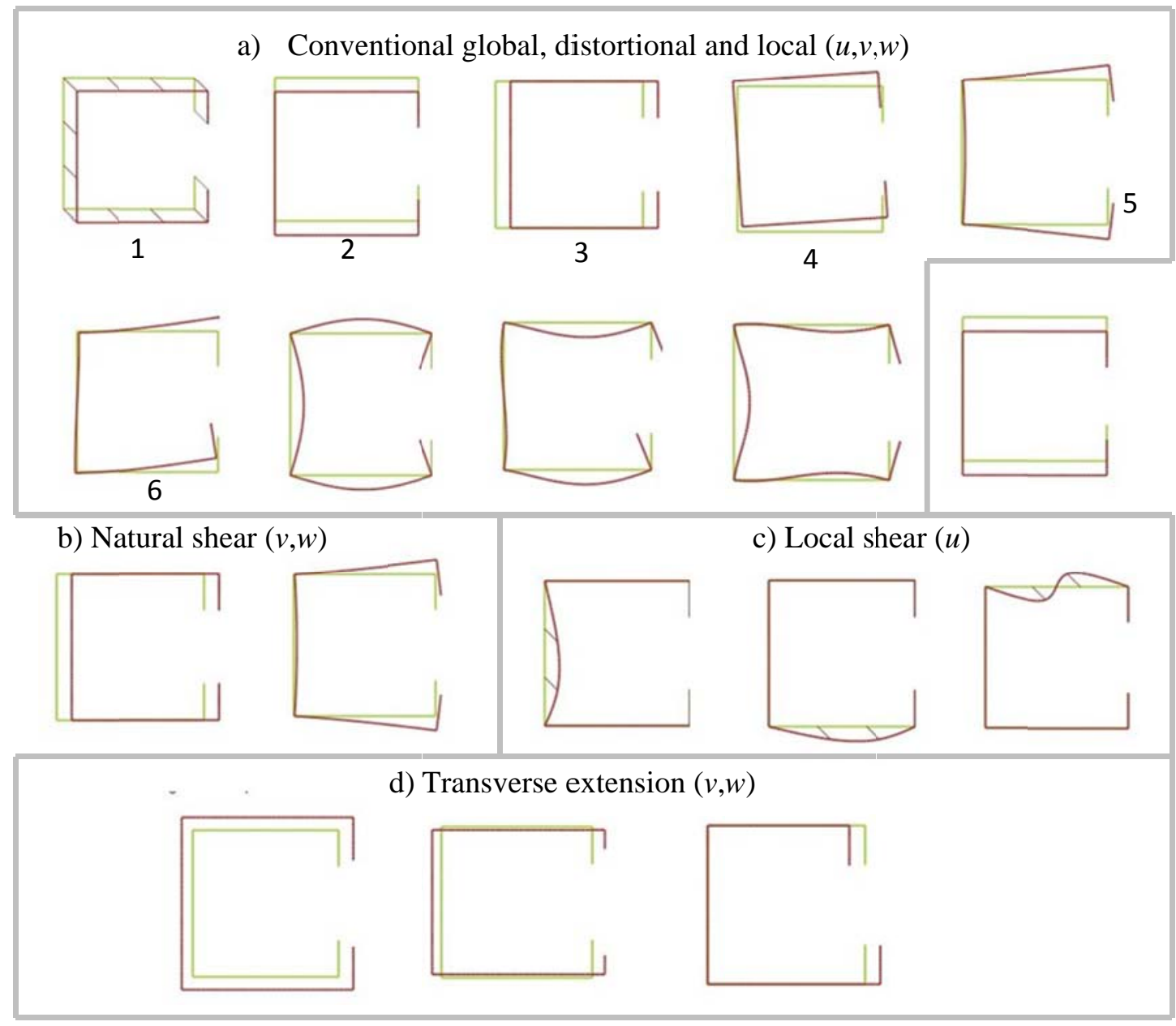

Fig. 2. Some of the GBT deformation modes of a channel cross-section (the non-null displacement components of each mode type have been included in brackets). 
In this modal approach, the deformation modes included in the analysis are selected depending on the complexity of the phenomenon to be simulated. For instance, when the global buckling load of a long member is calculated, only conventional global deformation modes are needed. On the contrary, when the effect of a concentrated load is studied, the analysis has to consider a larger number of modes, including, probably, conventional local, distortional and global modes, as well as some transverse-extension and local shear modes. However, it should be kept in mind that, in a similar way as in the standard finite element method, the more modes are considered in the analysis, the higher the computational cost is (although the GBT computational cost is usually far lower than the FEM computational cost).

In the GBT calculation procedure proposed herein for perforated members, the crosssection analysis is carried out twice, on the non-perforated and perforated cross-sections. In Fig. 3, it can be observed that both cross-sections are the same, but for the thickness of the perforated segment. All the analyses of this investigation are performed considering that the thickness of the perforated segment is $t / 100$, where $t$ is the gross sheet thickness. It should be pointed out that the use of this value of reduced thickness has only been verified for patterns of small rack perforations. Additional work should be carried out in the future to validate the use of the t/100 thickness in other types of perforations, such as in large and isolated perforations.

Fig. 3 also shows the cross-section discretization of a perforated channel member, where minimum two intermediate nodes are required for each hole.
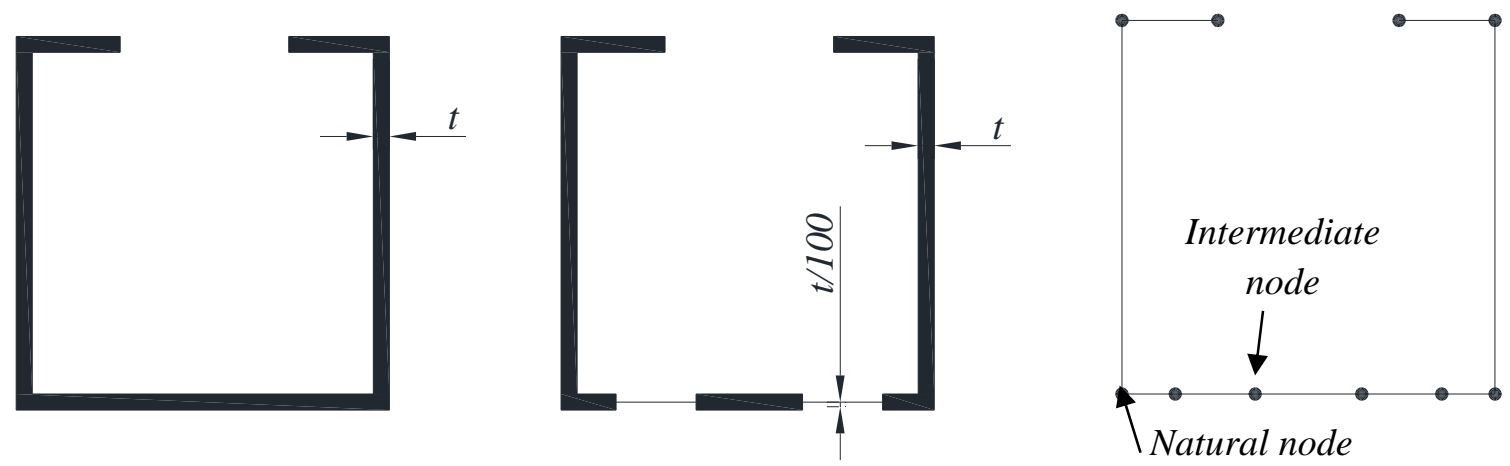

Fig. 3. Unperforated and perforated channel cross-section, and example of cross-section discretization.

Sub-indexes $n p$ and $p$ will be used for the parameters corresponding to the non-perforated and perforated cross-sections, respectively. For instance, the vectors of the deformation modes are designated as: $u_{k, n p}(s), v_{k, n p}(s)$ and $w_{k, n p}(s)$; and $u_{k, p}(s), v_{k, p}(s)$ and $w_{k, p}(s)$.

Finally, once the deformation modes have been determined, the displacement field of a member is expressed using a modal vector space:

$$
\begin{gathered}
u(x, s)=u_{k}(s) \phi_{k, x}(x), \\
v(x, s)=v_{k}(s) \phi_{k}(x), \\
w(x, s)=w_{k}(s) \phi_{k}(x),
\end{gathered}
$$

where the summation convention applies to subscript $k$, and $\phi_{k}(x)$ is the amplitude function of mode $k$ in the longitudinal member direction. 


\subsection{GBT Finite element stiffness matrices}

The aim of this Section is to introduce the stiffness matrices of a GBT based finite element, so that in the next Section it will be possible to easily demonstrate how these matrices are assembled.

The GBT finite element matrices are derived from the member strain energy variation $\delta U$ :

$$
\delta U=\delta U_{1}+\delta U_{2}
$$

where $\delta U_{1}$ is the variation of the strain energy first order terms ${ }^{14}$ :

$$
\delta U_{1}=\int_{L_{e}}\left(C_{i j} \cdot \phi_{i, x x} \cdot \delta \phi_{j, x x}+D_{i j} \cdot \phi_{i, x} \cdot \delta \phi_{j, x}+E_{i j} \cdot \phi_{i} \cdot \delta \phi_{j, x x}+E_{j i} \cdot \phi_{i, x x} \cdot \delta \phi_{j}+B_{i j} \cdot \phi_{i} \cdot \delta \phi_{j}\right) d x,
$$

and $\delta U_{2}$ is the variation of the strain energy second order terms. The second order term corresponding to the membrane longitudinal strain is the only one considered in the procedure proposed in this article:

$$
\delta U_{2}=\lambda \int_{L_{e}} W_{k}^{0} \cdot C_{k i j} \cdot \phi_{i, x} \cdot \delta \phi_{j, x} \cdot d x,
$$

In the equations above, $L_{e}$ is the length of the member (finite element), $\lambda$ is the load parameter; $W^{0}{ }_{k}$ are the stress resultants; and the other parameters are determined in the crosssection analysis: (i) the cross-section modal mechanical properties $C_{i j}, D_{i j}, E_{i j}$ and $B_{i j}$, and (ii) the geometric stiffness components $C_{k i j}$.

The stiffness matrices are obtained by introducing the proper $\phi_{k}(x)$ functions in equations (2) to (4) (see, for instance, Bebiano et al. ${ }^{15}$ ). The shape functions selected for the amplitudes of the conventional and natural shear modes are Hermite cubic polynomials (equation (5)); while Lagrange cubic polynomials (equation (6)) are used for the amplitudes of the local shear modes (Basaglia et al. ${ }^{16}$ ).

$$
\begin{aligned}
& \phi_{k}{ }^{H}(x)=\phi_{k, x}{ }^{H}(0) \cdot \psi_{1}{ }^{H}+\phi_{k}{ }^{H}(0) \cdot \psi_{2}{ }^{H}+\phi_{k, x}{ }^{H}\left(L_{e}\right) \psi_{3}{ }^{H}+\phi_{k}{ }^{H}\left(L_{e}\right) \cdot \psi_{4}{ }^{H} \\
& \psi_{1}{ }^{H}=L_{e}\left(\left(\frac{x}{L_{e}}\right)^{3}-2\left(\frac{x}{L_{e}}\right)^{2}+\frac{x}{L_{e}}\right) \quad \psi_{2}{ }^{H}=2\left(\frac{x}{L_{e}}\right)^{3}-3\left(\frac{x}{L_{e}}\right)^{2}+1 \\
& \psi_{3}{ }^{H}=L_{e}\left(\left(\frac{x}{L_{e}}\right)^{3}-\left(\frac{x}{L_{e}}\right)^{2}\right) \quad{\psi_{4}}^{H}=-2\left(\frac{x}{L_{e}}\right)^{3}+3\left(\frac{x}{L_{e}}\right)^{2}
\end{aligned}
$$




$$
\begin{aligned}
& \phi_{k, x}{ }^{L}(x)=\phi_{k, x}{ }^{L}(0) \cdot \psi_{1}{ }^{L}+\phi_{k, x}{ }^{L}\left(\frac{L_{e}}{3}\right) \cdot \psi_{2}{ }^{L}+\phi_{k, x}{ }^{L}\left(\frac{2 L_{e}}{3}\right) \cdot \psi_{3}{ }^{L}+\phi_{k, x}{ }^{L}\left(L_{e}\right) \cdot \psi_{4}{ }^{L} \\
& \psi_{1}{ }^{L}=\frac{\left(x-\frac{L_{e}}{3}\right)}{\left(0-\frac{L_{e}}{3}\right)} \cdot \frac{\left(x-\frac{2 L_{e}}{3}\right)}{\left(0-\frac{2 L_{e}}{3}\right)} \cdot \frac{\left(x-L_{e}\right)}{\left(0-L_{e}\right)} \quad \psi_{2}{ }^{L}=\frac{(x-0)}{\left(\frac{L_{e}}{3}-0\right)} \cdot \frac{\left(x-\frac{2 L_{e}}{3}\right)}{\left(\frac{L_{e}}{3}-\frac{2 L_{e}}{3}\right)} \cdot \frac{\left(x-L_{e}\right)}{\left(\frac{L_{e}}{3}-L_{e}\right)} \\
& \psi_{3}{ }^{L}=\frac{(x-0)}{\left(\frac{2 L_{e}}{3}-0\right)} \cdot \frac{\left(x-\frac{L_{e}}{3}\right)}{\left(\frac{2 L_{e}}{3}-\frac{L_{e}}{3}\right)} \cdot \frac{\left(x-L_{e}\right)}{\left(\frac{2 L_{e}}{3}-L_{e}\right)} \quad \psi_{4}{ }^{L}=\frac{(x-0)}{\left(L_{e}-0\right)} \cdot \frac{\left(x-\frac{L_{e}}{3}\right)}{\left(L_{e}-\frac{L_{e}}{3}\right)} \cdot \frac{\left(x-\frac{2 L_{e}}{3}\right)}{\left(L_{e}-\frac{2 L_{e}}{3}\right)}
\end{aligned}
$$

It is noted that Hermite polynomials are used in the present investigation for the axial extension mode, instead of the commonly used Lagrange polynomials. This is due to special requirements concerning the assembling process described in Section 2.3 and Section 3.2.

The final result of the stiffness matrix derivations is the usual finite element matrix equation (FEM linear buckling analysis equation):

$$
\left([K]^{(e)}+\lambda[G]^{(e)}\right)\{d\}^{(e)}=\{0\},
$$

where $[K]^{(e)}$ is the element stiffness matrix, $[G]^{(e)}$ is the geometric stiffness matrix, and $\{d\}^{(e)}$ is the displacement vector. The stiffness and geometric matrices have as many submatrices as the number of deformation modes considered in the analysis:

$$
\begin{gathered}
{[K]^{(e)}=\left[\begin{array}{ccc}
{\left[K^{11}\right]^{(e)}} & \cdots & {\left[K^{1 N}\right]^{(e)}} \\
\vdots & \ddots & \vdots \\
{\left[K^{N 1}\right]^{(e)}} & \cdots & {\left[K^{N N}\right]^{(e)}}
\end{array}\right],} \\
{[G]^{(e)}=\left[\begin{array}{ccc}
{\left[G^{11}\right]^{(e)}} & \cdots & {\left[G^{1 N}\right]^{(e)}} \\
\vdots & \ddots & \vdots \\
{\left[G^{N 1}\right]^{(e)}} & \cdots & {\left[G^{N N}\right]^{(e)}}
\end{array}\right] .}
\end{gathered}
$$

Similarly, the displacement vector is composed of a number of sub-vectors:

$$
\{d\}^{(e)}=\left\{\begin{array}{c}
\left\{d^{1}\right\}^{(e)} \\
\vdots \\
\left\{d^{N}\right\}^{(e)}
\end{array}\right\} .
$$

In the equations above, $N$ is the number of modes; $\left[K^{i j}\right]^{(e)}$ and $\left[G^{i j}\right]^{(e)}$ are $4 \times 4$ sub-matrices; and $\left\{d^{j}\right\}^{(e)}$ is also a 4-sized sub-vector. The components of the sub-matrices and sub-vector differ depending on the shape functions used for the amplitude $\phi_{k}(x)$, i.e., depending on whether $i$ and $j$ are Hermite or Lagrange modes. 
By having a look to the amplitude functions (5) and (6), it is easy to understand the components of sub-vectors $\left\{d^{j}\right\}^{(e)}$ in equation (10):

-Hermite modes:

$$
\left\{d^{j}\right\}^{(e), H}=\left\{\begin{array}{c}
\phi_{j, x}{ }^{H}(0) \\
\phi_{j}{ }^{H}(0) \\
\phi_{j, x}{ }^{H}\left(L_{e}\right) \\
{\phi_{j}{ }^{H}}^{H}\left(L_{e}\right)
\end{array}\right\} .
$$

-Lagrange modes:

$$
\left\{d^{j}\right\}^{(e), L}=\left\{\begin{array}{c}
\phi_{j, x}{ }^{L}(0) \\
\phi_{j, x}{ }^{L}\left(\frac{L_{e}}{3}\right) \\
\phi_{j, x}{ }^{L}\left(\frac{2 \cdot L_{e}}{3}\right) \\
\phi_{j, x}{ }^{L}\left(L_{e}\right)
\end{array}\right\},
$$

Finally, the expressions for the components of sub-matrices $\left[K^{i j}\right]^{(e)}$ and $\left[G^{i j}\right]^{(e)}$ are:

$$
\begin{gathered}
K_{p r}^{i j}=C_{i j} \int_{L_{e}} \psi_{p, x x}^{\alpha} \psi_{r, x x}^{\beta} d x+D_{i j} \int_{L_{e}} \psi_{p, x}{ }^{\alpha} \cdot \psi_{r, x}^{\beta} d x+E_{i j} \int_{L_{e}} \psi_{p}^{\alpha} \psi_{r, x x}^{\beta} d x+ \\
+E_{j i} \int_{L_{e}} \psi_{p, x x}^{\alpha} \cdot \psi_{r}^{\beta} d x+B_{i j} \int_{L_{e}} \psi_{p}^{\alpha} \psi_{r}^{\beta} d x \\
G_{p r}^{i j}=-W_{k}^{0} \cdot C_{k i j} \int_{L_{e}} \psi_{p, x}{ }^{\alpha} \psi_{r, x}{ }^{\beta} d x,
\end{gathered}
$$

where sub-indexes $p$ and $r$ range from 1 to 4 , and $\alpha$ and $\beta$ can be $H$ or $L$, depending on the $i$ and $j$ modes involved in the sub-matrix.

\subsection{Assembly of stiffness matrices}

Fig. 4(a) shows a perforated column discretized to create a GBT beam model. The member is composed of a series of non-perforated and perforated parts, and each part is discretized in a number of GBT finite elements (two elements per part in this figure). The cross-section in between two parts is called interface cross-section.

This Section illustrates how the global stiffness matrices of the GBT beam model are created:

$$
[K]=\left[\begin{array}{ccc}
{\left[K^{11}\right]} & \cdots & {\left[K^{1 N}\right]} \\
\vdots & \ddots & \vdots \\
{\left[K^{N 1}\right]} & \cdots & {\left[K^{N N}\right]}
\end{array}\right]
$$




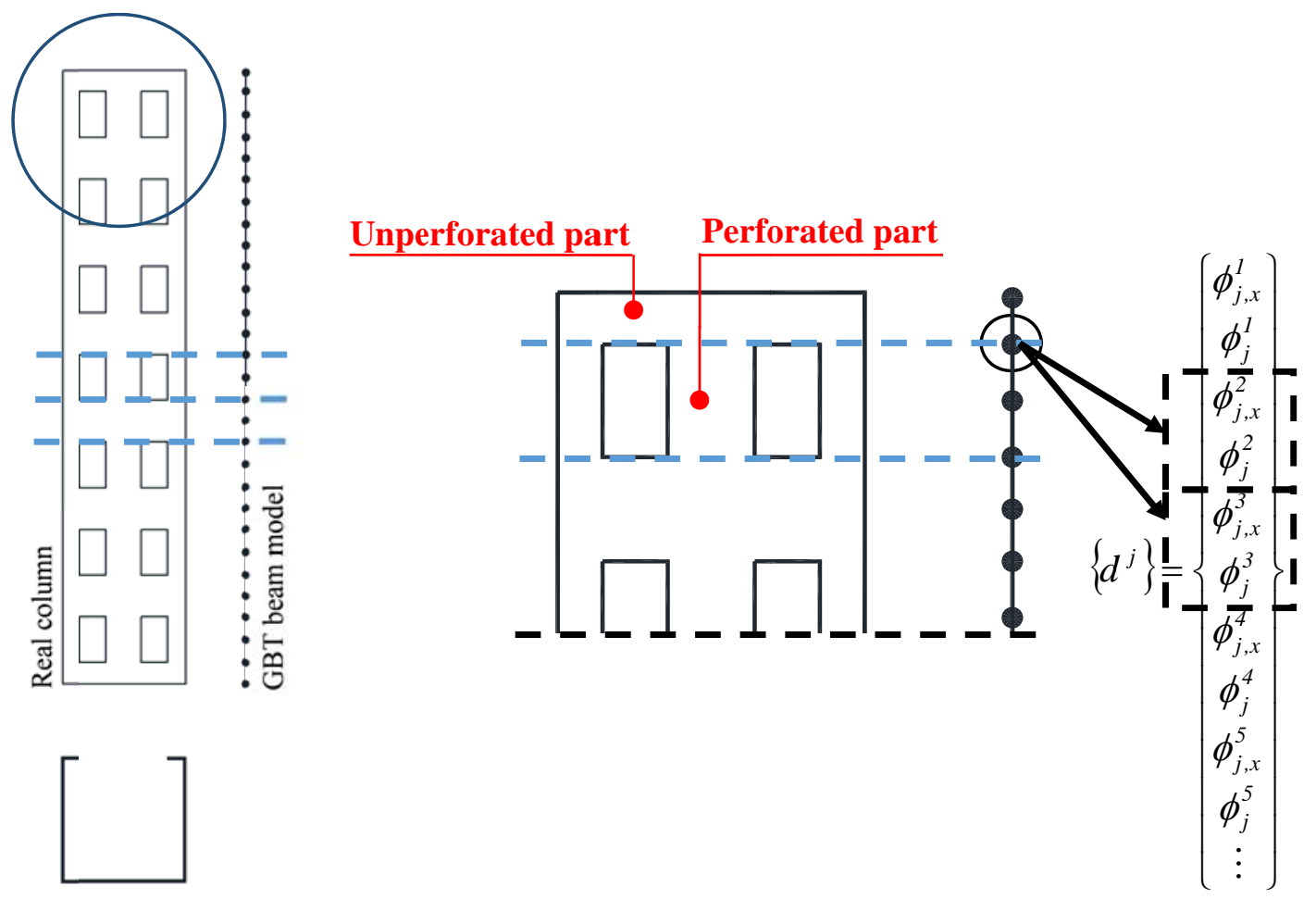

a) Degrees of freedom of coincident nodes to be linked by means of $\left[K^{i j}\right]=$ constraint equations

\begin{tabular}{|c|c|c|c|c|c|c|c|c|c|}
\hline$K_{11}^{i j, n p}$ & $K_{12}^{i j, n p}$ & $K_{13}^{i j, n p}$ & $\begin{array}{c}K_{14}^{i j, n p} \\
\end{array}$ & 0 & 0 & 0 & 0 & 0 & 0 \\
\hline$K_{21}^{i j, n p}$ & $K_{22}^{i j, n p}$ & $K_{23}^{i j, n p}$ & $K_{24}^{i j, n p}$ & 0 & 0 & 0 & 0 & 0 & 0 \\
\hline$K_{31}^{i j, n p}$ & $K_{32}^{i j, n p}$ & $K_{33}^{i j, n p}$ & $K_{34}^{i j, n p}$ & 0 & 0 & 0 & 0 & 0 & 0 \\
\hline$K_{41}^{i j, n p}$ & $K_{42}^{i j, n p}$ & $K_{43}^{i j, n p}$ & $K_{44}^{i j, n p}$ & 0 & 0 & 0 & 0 & 0 & 0 \\
\hline$\overline{0}$ & $\overline{0}$ & $\overline{0}^{-}$ & $-\overline{0}$ & $K_{11}^{i j, p}$ & $K_{12}^{i j j p}$ & $K_{13}^{i j, p}$ & $K_{14}^{i i j, i}$ & 0 & 0 \\
\hline 0 & 0 & 0 & 0 & $K_{21}^{i j, p}$ & $K_{22}^{i j, p}$ & $K_{23}^{i j, p}$ & $K_{24}^{i j, j}$ & 0 & 0 \\
\hline 0 & 0 & 0 & 0 & $K_{31}^{i j, p}$ & $K_{32}^{i j, p}$ & $K_{3, j}^{i, p}+K_{11}^{i j, p}$ & $K_{34}^{i j, p}+K_{12}^{i j, p}$ & $K_{13}^{i j, p}$ & $K_{14}^{i j, p}$ \\
\hline 0 & 0 & 0 & 0 & $K_{41}^{i j, p}$ & $K_{42}^{i j, p}$ & $K_{43}^{i j, p}+K_{21}^{i j, p}$ & $K_{44}^{i j, p}+K_{22}^{i j, p}$ & $K_{23}^{i j, p}$ & $K_{24}^{i j, p}$ \\
\hline 0 & 0 & 0 & 0 & 0 & 0 & $K_{31}^{i j, p}$ & $K_{32}^{i j, p}$ & $K_{33}^{i j, p}$ & $K_{34}^{i j, p}$ \\
\hline 0 & 0 & 0 & 0 & 0 & 0 & $K_{41}^{i j, p}$ & $K_{42}^{i j, p}$ & $K_{43}^{i j, p}$ & $K_{44}^{i j, p}$ \\
\hline
\end{tabular}

b) Assembled stiffness matrices of elements 1, 2 and 3. The dashed rectangles are connected by means of constraint equations

Fig. 4. Assembly of the global stiffness matrix. 
$[G]=\left[\begin{array}{ccc}{\left[G^{11}\right]} & \cdots & {\left[G^{1 N}\right]} \\ \vdots & \ddots & \vdots \\ {\left[G^{N 1}\right]} & \cdots & {\left[G^{N N}\right]}\end{array}\right]$.

The explanation focuses on one of the $\left[K^{i j}\right]$ sub-matrices. The other sub-matrices of the global stiffness matrix and global geometric stiffness matrix are generated in a similar way.

The first step is the finite element assembly inside each part. It follows the standard GBT finite element assembly procedure, where finite elements are connected one to each other by simply locating the element stiffness matrices in the proper place. This is described in Fig. 4(b) (dotted rectangles) for the first three elements of the model in Fig. 4(a). The modes involved in the sub-matrix of this figure are supposed of Hermite type.

The second step is the assembly of the parts, which can also be described from Fig. 4. The dashed rectangles in this figure contain the degrees of freedom, and sub-matrices, to be linked. It is worth noting that at the interface between two parts there are two coincident nodes, and sets of degrees of freedom. The connection between the parts is established by means of constraint equations on the degrees of freedom of each coincident node, i.e., on the amplitude values at the interface. Constraint equations are used to reduce the duplicated set of degrees of freedom to a single set. This is achieved by imposing continuity of displacements in the $u-v-w$ space at different points of the cross-section.

Two different sets of constraint equations per connection should be defined for Hermite modes, since degrees of freedom $\phi_{k}^{l}$ and $\phi_{k, x}^{l}$ are present in the displacement vector; while just one set of equations is needed for Lagrange modes, because they only have $\phi_{k, x}^{l}$ terms. An example of constraint equations can be:

-Hermite modes:

$$
\begin{aligned}
& u_{l}(x, s)-u_{l+1}(x, s)=u_{k, n p}(s) \phi_{k, x}^{l}-u_{k, p}(s) \phi_{k, x}^{l+1}=0, \\
& w_{l}(x, s)-w_{l+1}(x, s)=w_{k, n p}(s) \phi_{k}^{l}-w_{k, p}(s) \phi_{k}^{l+1}=0,
\end{aligned}
$$

-Lagrange mode:

$$
u_{l}(x, s)-u_{l+1}(x, s)=u_{k, n p}(s) \phi_{k, x}^{l}-u_{k, p}(s) \phi_{k, x}^{l+1}=0,
$$

where summation convention applies to $k$; and $\phi_{k}^{l}, \phi_{k, x}^{l}, \phi^{l+1}{ }_{k}$ and $\phi^{l+1}{ }_{k, x}$ are the degrees of freedom of the coincident nodes $l$ and $l+1$ (see equations (11) or (12)). Node $l$ can belong to the cross-section of the non-perforated part, and node $l+1$ to the cross-section of the perforated part (or vice versa).

At this point, it is important to note that equations (16) are a first proposal, introduced here to explain the general analysis procedure. They are the constraints that anyone familiar with GBT would probably choose as a first option to solve the problem of connection between parts. However, it will be seen that this set of constraint equations may lead to a final nonrobust procedure. Consequently, in the end, a different option will be chosen. This issue is discussed in Section 3.2, together with complementary explanations on: which degrees of freedom ( $u, v$ or $w$ ) should be considered in the equations; and how these equations should be applied to constrain (link) different points of the coincident cross-sections.

The constraints are finally introduced into the finite element procedure via the Lagrange multiplier's method. This involves the generation of a constraint matrix $[C]$, which is combined with the stiffness matrix in the following way: 


$$
\left[K_{c}\right]=\left[\begin{array}{lc}
{[K]} & {[C]^{T}} \\
{[C]} & {[0]}
\end{array}\right],
$$

where $\left[K_{c}\right]$ is the constrained global stiffness matrix; $[K]$ is the global stiffness matrix presented above (equation(15.a)); and [0] is a matrix containing zeros. The geometric stiffness matrix $\left[G_{c}\right]$ can be obtained in a similar way. Finally, the eigenvalue problem to be solved to determine the elastic bucking loads and modes can be constructed:

$$
\left(\left[K_{c}\right]+\lambda\left[G_{c}\right]\right)\left\{d_{c}\right\}=\{0\} .
$$

\section{VALIDATION AND ILLUSTRATIVE EXAMPLES}

\subsection{Introduction to the examples}

So far, the article has presented a general outline of the proposed GBT procedure for perforated columns. There are still some important issues to be discussed that will be introduced in this Section by means of examples. The validation of the procedure is also presented here step by step as the different issues are solved.

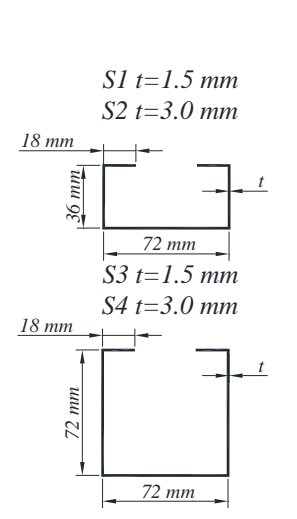

a) Channel rack columns (S1 to S4)

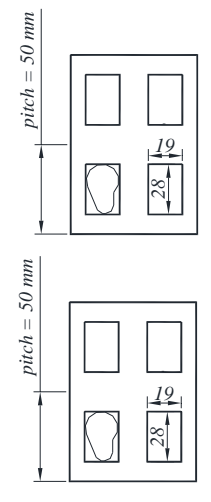

Fig. 5. Investigated columns.

Fig. 5 shows the columns considered in the examples and final validation: two channel columns, and two omega columns. In all cases, two different sheet thicknesses have been considered: $1.5 \mathrm{~mm}$ and $3 \mathrm{~mm}$, which correspond to the lower and upper limits of the range of thickness commonly used for such columns in pallet rack structures.

The channel columns are similar to those normally produced by North American rack manufacturers, showing the typical tear-drop holes. On the other hand, the omega crosssections are mostly used in Europe and Australia, and they can show different hole shapes and sizes. Each manufacturer has its own hole configuration. The configurations chosen in this study for the omega cross-sections are similar to those of real rack columns.

However, it should be underlined that only rectangular holes are allowed in the proposed method. Consequently, the analyses are performed on columns containing rectangular holes that are large enough to circumscribe the real holes (Fig. 5). Another simplification is that only web holes are included in the column models, although most of the rack columns show web and flange holes. If the flange holes had been included, the resulting GBT beam model would have been too complex for a first verification of the procedure. Nevertheless, it should 
be pointed out that more realistic column models, including non-rectangular holes and flange perforations, have been recently tested with satisfactory results in Casafont et al. ${ }^{17}$.

In addition to the perforation patterns shown in Fig. 5, the proposed procedure has also been tested on the patterns included in Fig. 6.

\section{Perforation patterns for S1 to S4 columns}
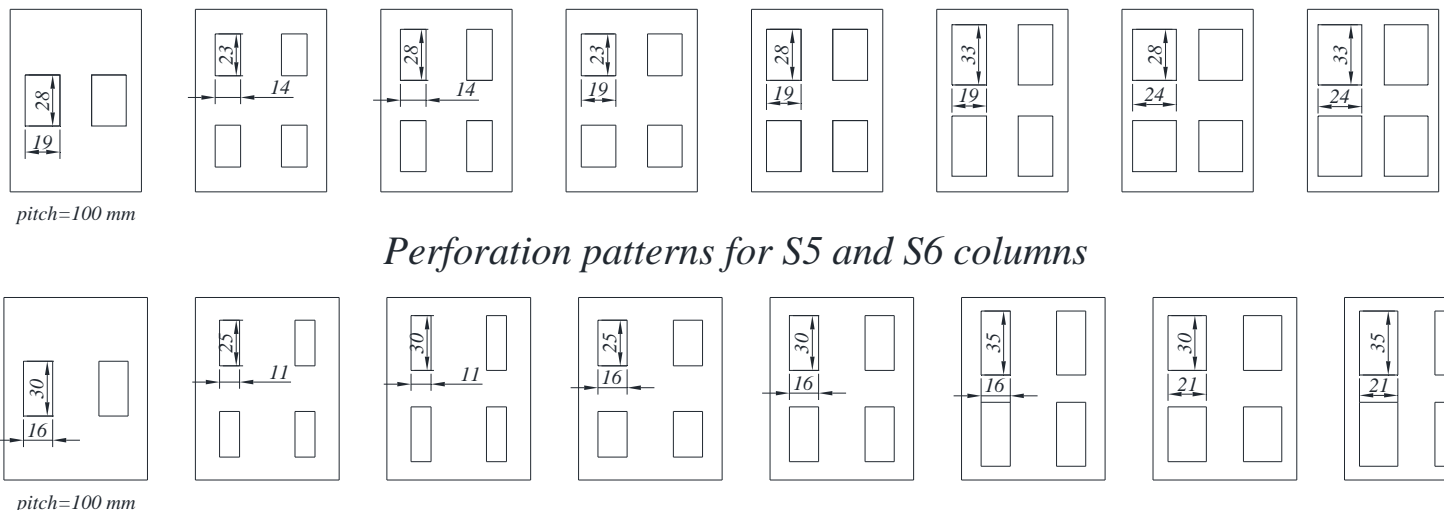

Perforation patterns for S5 and S6 columns
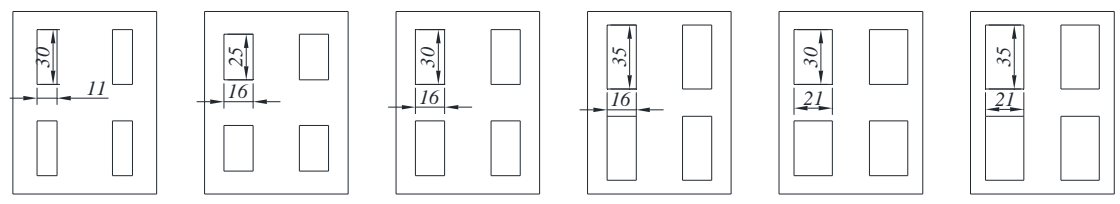

Perforation patterns for S7 and S8 columns
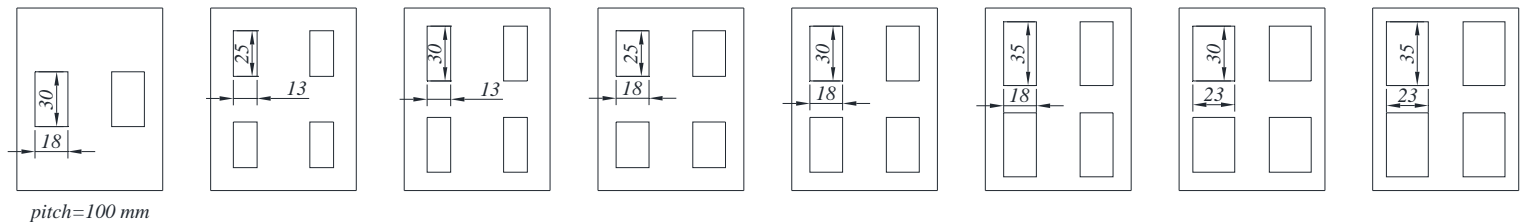

Fig. 6. Investigated perforation patterns. The pitch is $50 \mathrm{~mm}$, except where indicated. (dimensions in $\mathrm{mm}$.)

Most of the discussions in the following Sections of the article are illustrated by means of analyses on a fixed ended S4 column (Fig. 5(a)). However, all columns in Figs. 5 and 6, pinned and fixed ended, are used to validate the accuracy of the different proposed procedures.

The discussions are mainly focused on the following issues: (i) the performance of constraint equations (16); (ii) the selection of the deformation modes to be included in the analyses; and (iii) the sensitivity of the results to the cross-section and member discretization. The objective is to use the minimum number of constraint equations and deformation modes, and the simplest discretization in order to reduce the computational cost as much as possible. It is worth noting that, since the discretization has to be adapted to a large number of holes, the number of degrees of freedom can be very large.

A program in Matlab ${ }^{18}$ has been developed to carry out the analyses with the proposed GBT procedure. The validation of the procedure is verified by running this program multiple times for different member cross-sections and lengths, and comparing the resulting buckling loads to the results of analyses performed with shell finite elements (SFEM). The finite element work has been done in ANSYS ${ }^{19}$.

The present Section closes with Fig. 7 that shows the buckling load curves of column S4 with and without holes obtained via SFEM. It can be observed that the effect of the holes is significant, and should be considered in the analyses. In this figure, the L curve represents local buckling loads, SD symmetric distortional buckling loads, AD anti-symmetric distortional buckling loads, TF torsional-flexural buckling loads, and $\mathrm{F}$ flexural buckling loads. The curves correspond to buckling loads of dominant buckling modes identified by 
visual inspection of the deformed shapes that result from the analysis. For instance, the SD curve corresponds to a buckling mode where the symmetric distortional buckling mode is dominant.

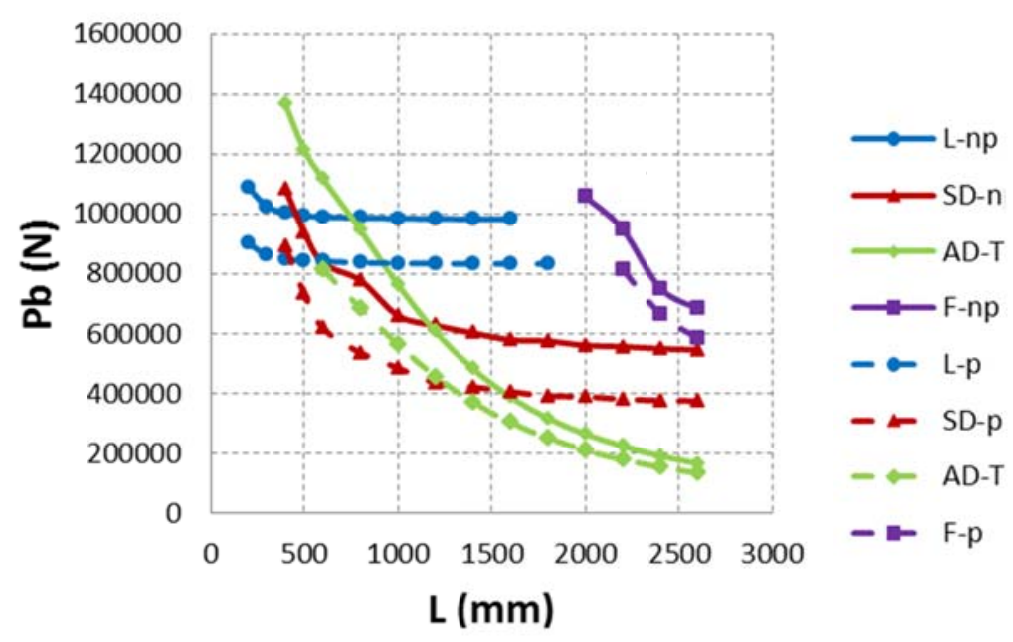

Fig. 7. Elastic buckling loads of FEM perforated (p) vs. FEM non-perforated (np) for S4 column.

\subsection{Selection of constraint equations}

The first steps of the investigation were devoted to study how equations (16) had to be used: number of constraint equations to be applied, and modal displacement components involved. The problem is that a wrong decision on these two points (and others that will be seen next) can lead to a non-robust analysis procedure. It is very important to avoid: (i) singular or ill conditioned matrices in the final eigenvalue problem, equation (18); and (ii) non-meaningful results, i.e., unrealistic buckling modes or excessively non-accurate buckling loads.

The decision on the constraint equations was made by testing different alternatives until a robust option was found. The authors started working with equations (16), but in the end it was verified that there are better options, as it will be seen later on in this Section. The aim of the following paragraphs is to reflect the work done to solve this issue.

The first analyses applied constraint equations as defined in (16), considering only distortional and global deformation modes. From the beginning, it was decided to set a number of constraint equations equal to the number of degrees of freedom to be linked. For instance, column S4 has four global modes and two distortional modes. Consequently, if the analyses are carried out considering these 6 modes, it is necessary to define 12 constraint equations per each set of connected cross-sections: six of type (16a) for the amplitude of the displacement derivative, $\phi_{k, x}^{l}$; and 6 of type (16b) for the amplitude of the displacement, $\phi_{k}^{l}$. If the axial deformation mode is considered Lagrangian, the number of (16b) equations becomes 5 .

Actually, in S4 there are 24 degrees of freedom at each interface, because there are 12 degrees of freedom for each one of the parts to be linked. The role of the constraint equations is to reduce the 24 degrees of freedom to 12 , which is the number of degrees of freedom of "conventional" cross-sections within each part.

In (16), continuity of $u$ and $w$ is forced to set the equations, while the $v$ component is not considered. If continuity of $v$ had also been imposed in the analysis, 6 constraint equations on $\phi^{l}{ }_{k}$ would have been added to the 6 already defined to ensure the continuity in $w$ (see equations (1) and (16)). The problem with the additional equations on $v$ is that they are causing the matrices of the eigenvalue problem (18) to be ill conditioned, which results in 
final incoherent results (if it is wanted to force continuity in $v$, then it is necessary to remove continuity equations on $w$ ). This is the main reason why it is recommended not to overconstrain the duplicated degrees of freedom at the connection.

From the discussion of the previous paragraph, it can also be concluded that in the proposed approach is not possible to enforce displacement continuity conditions in the usual manner. For example, it is not possible to enforce full continuity of the $u, v$ and $w$ displacements and their derivatives. In this sense, it has to be recalled that the actual GBT degrees of freedom of the system are $\phi_{k}^{l}$ and $\phi_{k, x}^{l}$ (amplitude and amplitude of the derivative), and that it would have made sense to force their continuity at the connection. Equations (16) are, in fact, introducing such continuity condition, but in a special way: instead of forcing the continuity separately mode by mode, it is forced combining modes. The combination of modes is introduced with mechanical sense, considering continuity conditions in the $u-v-w$ space. This was done in this way since the beginning following references by Basaglia et al. $^{20,21}$, which deal with the modelling of joints in GBT. Actually, the authors tested the option of imposing continuity of $\phi_{k, x}^{l}$ and $\phi^{l}{ }_{k}$ mode by mode, but the results were not satisfactory. The problem is that the number of GBT degrees of freedom at the interface between parts is usually lower than the number of $u, v$ and $w$ components at the connecting points. Consequently, it is not possible to force full $u-v-w$ continuity without overconstraining the GBT degrees of freedom. Nevertheless, it will be seen bellow that it is possible to produce buckling modes showing a continuous and smooth shape, and reasonable good estimations of buckling loads.

The equations are set by selecting a number of connecting points, i.e., $s$ values in equations (16) (see parameter $s$ in Fig. 8(a)), so that the total number of required constraint equations can be defined. In the present article, the connecting points are located at the natural nodes and at the mid-point of the cross-section segments. For instance, in the first steps of the investigation, and as a first trial, the 6 connecting points shown in Fig. 8(b) were used for cross-section S4. This allowed the procedure to set the 12 constraint equations discussed in the previous paragraphs. When the axial deformation mode was considered Lagrangian, only 5 connecting points were selected for equation (16b), the $w$ connecting point between nodes 8 and 9 was eliminated.

The location of the connecting points is a matter of study. There are sets of connecting points that can produce singular or ill conditioned systems, in spite of using the right number of equations. At the end of this Section, a set of connecting points that has been fully tested by the authors during the investigation is recommended.

In conclusion, it can be seen that there are different options to set the constraint equations, but not all possible options are valid. Different combinations of equations were tested in the present investigation. A few of them are reported in the following paragraphs to show some of the problems encountered when developing the analysis procedure.

Fig. 9(e) displays the critical buckling loads resulting from the first GBT analysis of column S4 (6u5w values). It was performed applying constraint equations (16), with 6 deformation modes and 6 connecting points (Fig. 8(b)). The axial mode was considered Lagrangian (only $5 \mathrm{w}$-continuity equations were applied). The results are rather low when compared to the shell finite element loads (SFEM values). Displays of the critical buckling mode for a $1400 \mathrm{~mm}$ long column can be seen in Fig. 9(a). It can be observed that the perforated and non-perforated parts are poorly connected. 


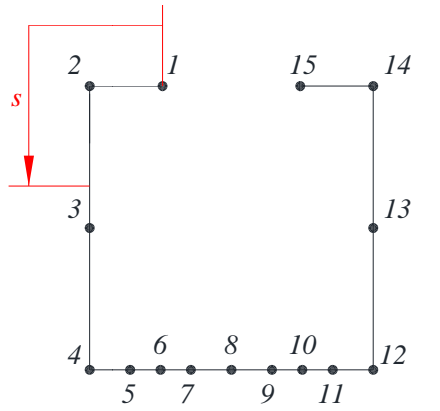

a)

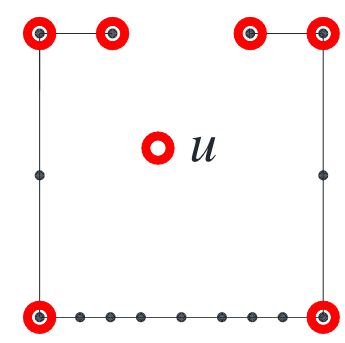

c)

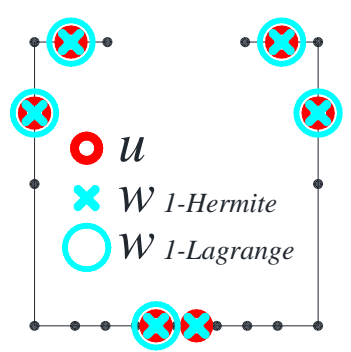

b)

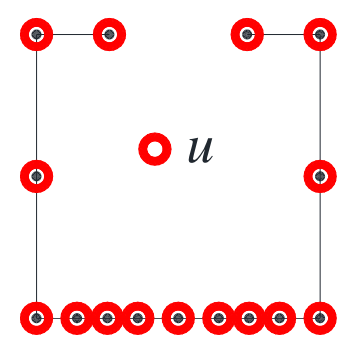

d)

Fig. 8. Cross-section discretization and connecting points considered in the initial studies.

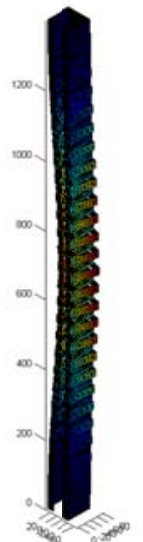

a) $6 u 6 \mathrm{w}$

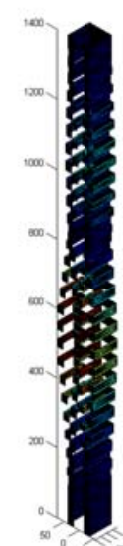

b) $6 u 3 w 2 v$

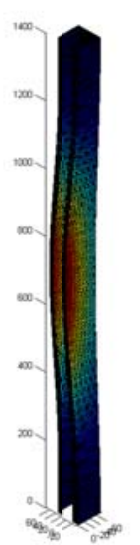

c) $6 u 6 u$

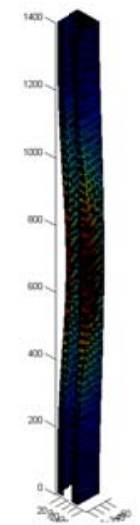

d) $6 u 5 u$

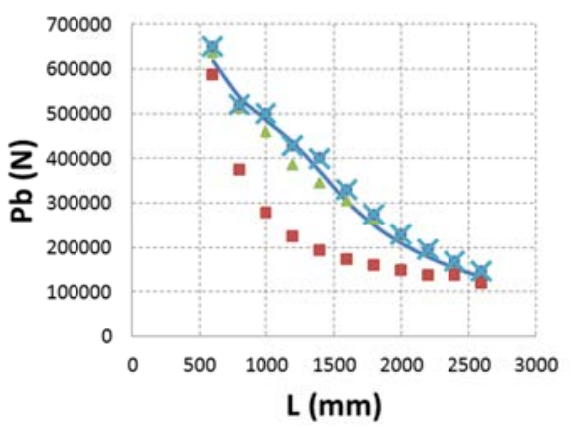

e) Critical buckling loads

-SFEM

- $6 \mathrm{u} 5 \mathrm{w}$-Fig.8(b)

A $6 u 3 w 2 v-F i g .8(b)$

- 6u6u-Fig.8(c)

$\times 6 u 5 u-F i g .8(c)$

Fig. 9. First results for column S4 obtained considering distortional and global deformation modes.

It was decided to improve the results by adding new constraints to the model. The aim was to increase the number of links at the interface between parts in order to improve the buckling mode shape and increase the critical buckling load. Consequently, the following constraint equations were added:

$$
v_{l}(x, s)-v_{l+1}(x, s)=v_{k, n p}(s) \phi_{k}^{l}-v_{k, p}(s) \phi_{k}^{l+1}=0,
$$

The equations were also applied to the five connection points shown in Fig. 8(b), resulting in 16 constraint equations for 11 degrees of freedom per interface. As expected, according to what has been pointed out above, the eigenvalue system became ill conditioned and no coherent solution was obtained (the eigenvectors and eigenvalues were complex and had no 
physical meaning). Then, analyses combining 6 constraint equations of type (16a) plus 3 equations of type (16b) and 2 equations of type (19) were carried out. Fig. 9 also shows the results when using the connecting points of Fig. 8(b) (two of the $w$ connecting points in this figure were used as $v$ connecting points). Now the system could be satisfactorily solved, the buckling loads improved, $6 u 3 w 2 v$ values, but the shape of the buckling modes was not correct (Fig. 9(b)).

After testing different constraint equations, it was verified that the best results are obtained with:

$$
\begin{gathered}
u_{k, n p}(s) \phi_{k, x}^{l}-u_{k, p}(s) \phi_{k, x}^{l+1}=0, \\
u_{k, n p}(s) \phi_{k}^{l}-u_{k, p}(s) \phi_{k}^{l+1}=0,
\end{gathered}
$$

All constraints were applied to the natural nodes (Fig. 8(c)). It should be pointed out that these nodes were not used to set the constraint equations in the previous analyses, where $u-w$ continuity is imposed. This is due to the fact the $w$ component has two different values in the natural nodes, depending on which element adjacent to the node is considered.

The first equation in (20) is (16a), while in the second one the modal longitudinal displacements $\left(u_{k, n p}\right.$ and $\left.u_{k, p}\right)$ are used instead the transverse displacements ( $w_{k, n p}$ and $\left.w_{k, p}\right)$. The use of $u_{k}(s)$ components combined with $\phi_{k}{ }_{k}$ is consistent in GBT, due to the existing linear relationships between $w_{k}(s)$ nodal values of the conventional modes and the $u_{k}(s)$ nodal values $^{9,12,13}$. The resulting buckling load curves and buckling mode shapes are rather good, as shown in Figs. 9(c) to 9(e). See also Fig. 10(a), that shows the $\mathrm{P}_{\mathrm{b}, \mathrm{GBT}} / \mathrm{P}_{\mathrm{b}, \mathrm{SFEM}}$ ratios corresponding to the analyses presented so far. It is noted that two different sets of values are included in this figure: the $6 u 6 u$ values correspond to analyses carried out considering that the axial mode is Hermitian, while the $6 u 5 u$ values are obtained from analyses; where this mode is considered Lagrangian. In this last case, since one of the (20.b) equations is removed, a connecting point has also to be removed from Fig. 8(c). The (20.b) equation corresponding to node 14 (Fig. 8(a)) is the equation chosen to be removed from the constraining set (i.e., the equation (20.b) that is set using the $s$ value corresponding to node 14 is removed). It can be observed that both analyses produce almost the same buckling loads (see allso Fig. 10(a)).

As pointed out in Section 2.2, the axial mode was finally considered Hermitian to avoid the need of deciding which natural node should be unselected when defining the constraint equations.

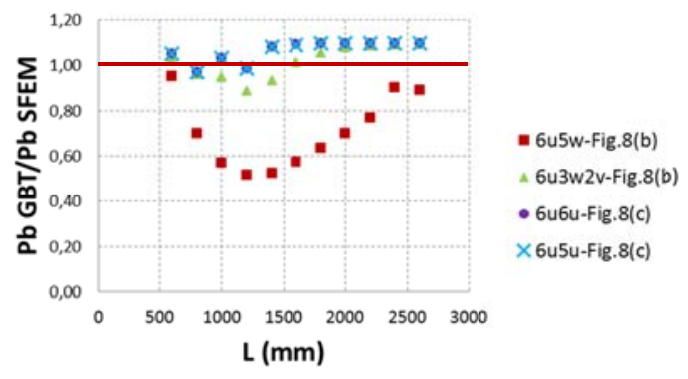

a) Analysis with DG modes

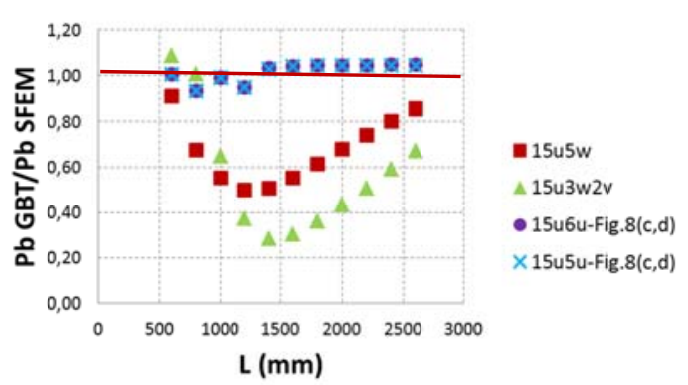

b) Analysis with DG and LS modes

Fig. 10. Accuracy of the first analyses carried out for column S4.

Similar calculations were carried out adding the local shear modes and the corresponding constraint equations and connecting points. When global, distortional and local shear modes are considered, constraint equations (20) transform into: 


$$
\begin{gathered}
u_{k, n p}^{D G}(s) \phi_{k, x}^{l}-u_{k, p}^{D G}(s) \phi_{k, x}^{l+1}+u_{k, n p}^{L S}(s) \phi_{k, x}^{l}-u_{k, p}^{L S}(s) \phi_{k, x}^{l+1}=0, \\
u_{k, n p}^{D G}(s) \phi_{k}^{l}-u_{k, p}^{D G}(s) \phi_{k}^{l+1}=0,
\end{gathered}
$$

where DG and LS denote the family of distortional and global deformation modes, and the family of local shear modes, respectively. Since local shear modes are of Lagrange type, equation (20.b), and its corresponding connecting points (Fig. 8(c)), do not change. The connecting points for equation (21a) are plotted in Fig. 8(d). After these analyses, the conclusion was the same (Fig. 10(b)): the best option is to use constraint equations similar to (20) and connecting points in Fig. 8(c), i.e., in this case, equations (21) with connecting points Fig. 8(c,d).

Table 1 shows the constraint equations and connecting points that are finally chosen for each family of deformation modes. The constraint configurations included in the Table have been defined so that the number of constraint equations is equal, or very similar, to the number of degrees of freedom. By proceeding in this way, it is ensured a final robust procedure, and it is avoided the occurrence of ill conditioned systems producing incoherent results.

Table 1. Recommended constraint equations and connecting points

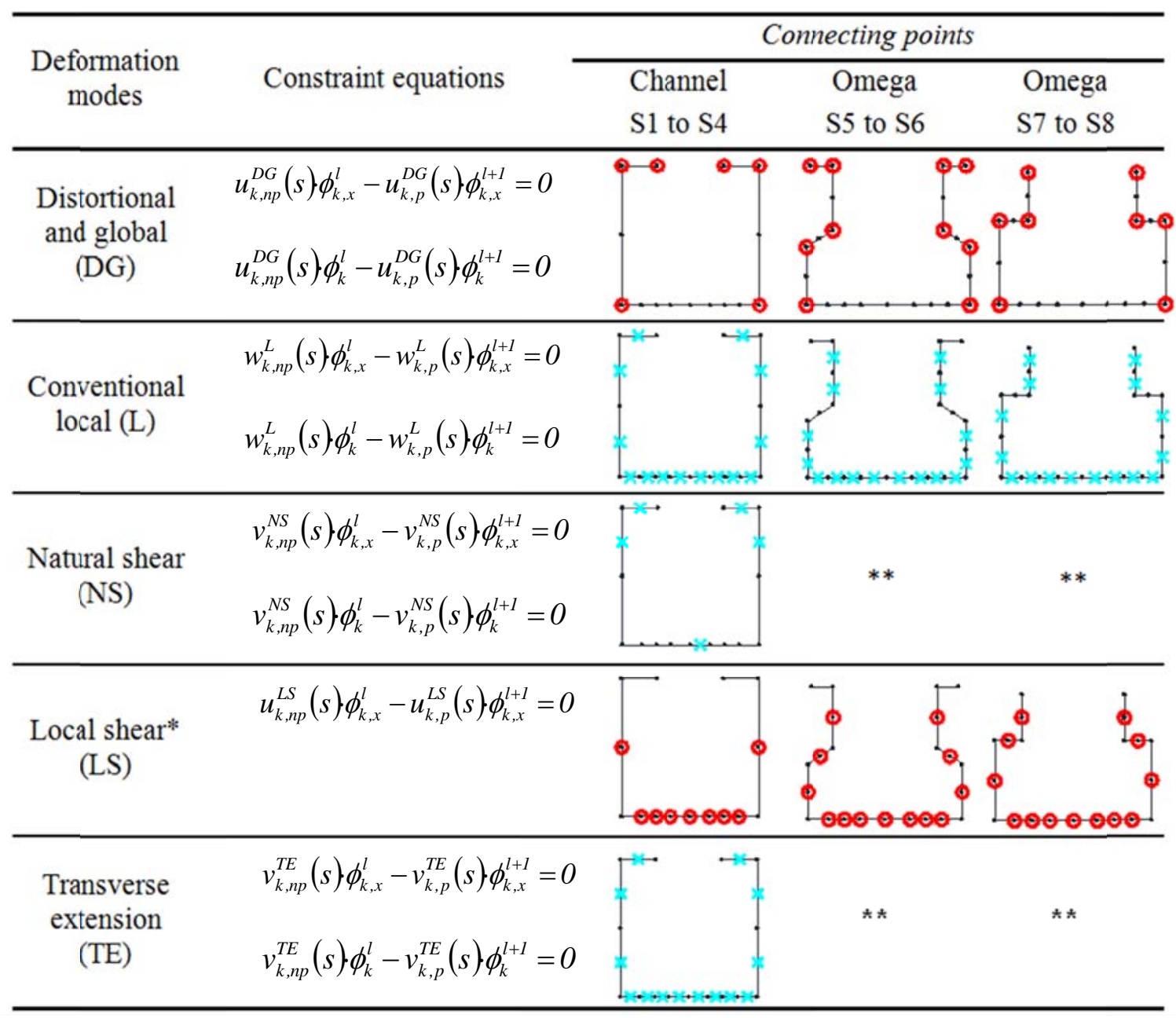

* The LS constraint equations and connecting points are added to the DG constraint equations and connecting points as described in eq. (21.a) and Fig. 8(d), respectively.

** NS and TS constraint equations for omega columns have not been investigated yet. 
For some mode families, the condition mentioned in the previous paragraph cannot be applied without a decision on the cross-section nodes or segments to be constrained. An example can be found when working with conventional local modes, for which the number of cross-section segments is always higher than the number of deformation modes. If, for instance, such modes are included in the analysis of the S4 column, using the discretization shown in Table 1, 11 constraint equations have to be added because there are 11 new duplicated degrees of freedom at the interface cross-sections. Introducing 11 constraint equations, involves unselecting 3 of the 14 segments of the discretized cross-section. To avoid making a decision on the segments to be eliminated, it was decided to constrain all segments. Although the condition on the number of constraint equations is not accomplished, it has been verified that the method keeps on being robust for the tested channel cross-sections.

Another situation where additional segments are constrained is when a symmetric pattern of connecting points is wanted. This is the case of the omega cross-section local modes in Table 1. For example, in cross-sections S5 and S6, the number of conventional local deformation modes is 15 , so 15 segments have to be constrained. However, in the end, 16 segments are constrained in order to generate a symmetric pattern of connecting points. As a consequence, no decision has to be made concerning the unpaired constraint equation (connecting point). It is also worth to point out that in the case of omega columns it is not recommended to constrain all the cross-section segments, as it was done for simple channel cross-sections. Constraining all the segments produce excessively high local buckling loads.

\subsection{Calculation of initial stresses}

Before going deeper into the study of the buckling load calculation, it is worth devoting a short section of the article to the initial stress resultants (GBT internal forces), that are used to generate the geometric stiffness matrices.

The initial stress resultants are determined from the displacement vector calculated by solving the following equation:

$$
\left[K_{c}\right]\left\{d_{c}\right\}=\{F\}
$$

where $\{F\}$ is the vector of external loads. Since members under pure compression are only investigated in the present study, the force vector has a unitary compression force at the top node of the column (on the $\phi_{1, x}$ degree of freedom), and the other components are zero.

The stress resultants $W^{0}{ }_{k}$ in equations (4) and (14) are derived from the solution of (22). It is worth to note that they are not limited to a pure compression. The presence of holes produces secondary internal forces that are considered in the analysis. For instance, the compression load causes a secondary bending moment (corresponding to mode 3 in Fig. 2), because the centroid of the perforated part of the column is different from the centroid of the non-perforated part. In a similar way, there are other initial stress resultants associated to other deformation modes.

It is interesting to study the stresses produced by the GBT internal forces. This allowed the authors to assess the performance of the proposed procedure when carrying out linear analyses, and to identify which stress components had to be considered in the generation of the geometric stiffness matrix. The aim was to include only the most significant stresses in the analysis.

First, the initial $\sigma_{\mathrm{x}}, \sigma_{\mathrm{s}}$ and $\sigma_{\mathrm{xs}}$ SFEM stresses are compared in Figs. 11(b) and 11(c), that show the values corresponding to the web of the cross-sections indicated in Fig. 11(a). The stress values are taken from the outer plane of the sheet, and have been normalized to the 
maximum $\sigma_{x}$ stress. Similar stress distributions can be observed in the other planes within the thickness of the sheet because it is mainly subjected to pure compression (the plate bending and torsional stress components are small). It can be concluded from these graphs that longitudinal stresses are significantly higher than the others. The maximum value of the transverse stress $\sigma_{\mathrm{s}}$ is about $20 \%$ of the maximum longitudinal stress $\sigma_{\mathrm{x}}$, and shear stresses $\sigma_{\mathrm{xs}}$ are only relevant in the cross-section near the perforations.

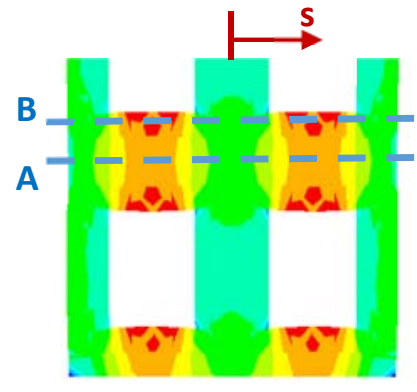

a) $\sigma_{x}$ web stress

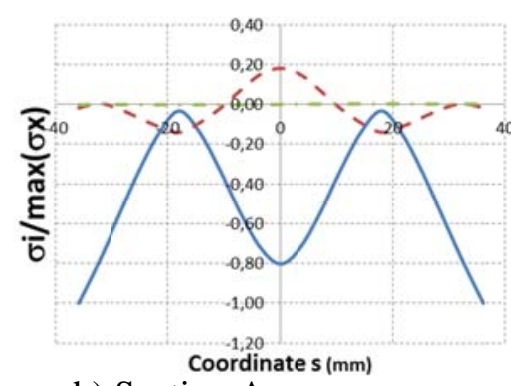

b) Section A

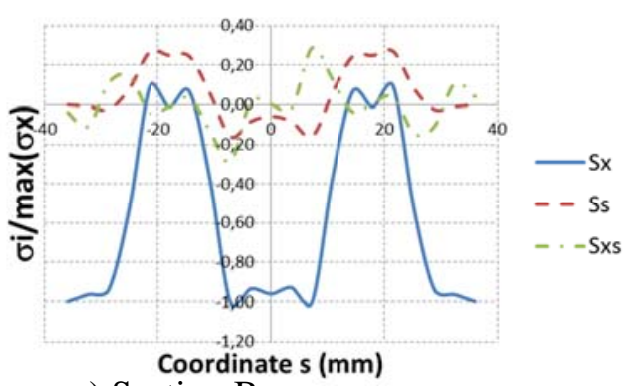

c) Section B

Fig. 11. Normalized SFEM stresses (stresses are shown normalized with respect to the maximum stress value).

Fig. 12 shows the stress distribution at the same cross-sections calculated by means of the GBT proposed procedure. The results are very good for the longitudinal stresses in terms of distribution and magnitude. On the other hand, the level of stresses corresponding to $\sigma_{\mathrm{s}}$ and $\sigma_{\mathrm{xs}}$ is in agreement with the SFEM results, but it is not possible to match the exact values in some cases: $\sigma_{\mathrm{s}}$ in Section A, and $\sigma_{\mathrm{xs}}$ in Section B.

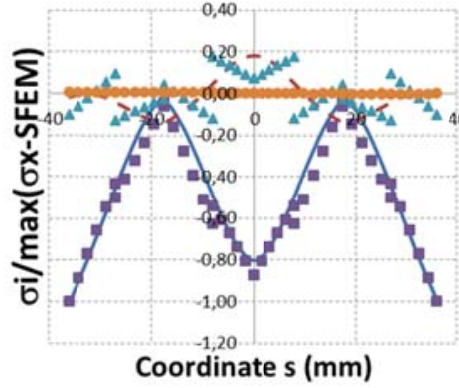

a) Section $\mathrm{A}$

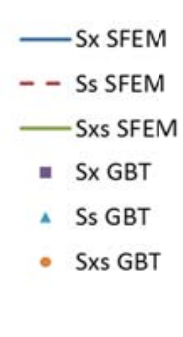

Fig. 12. SFEM stresses vs. GBT stresses.

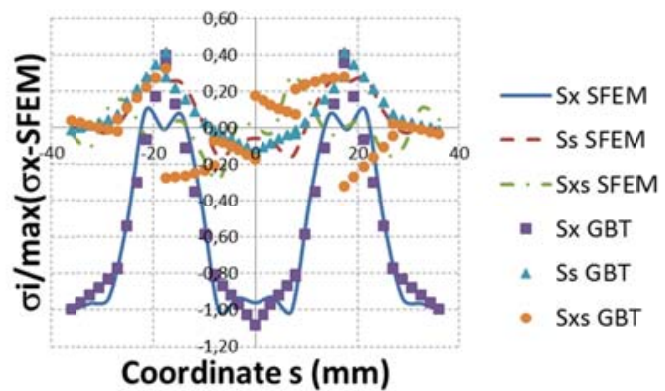

b) Section B

The focus of the study was then put on the longitudinal stresses $\sigma_{\mathrm{x}}$. The other components, $\sigma_{\mathrm{s}}$ and $\sigma_{\mathrm{xs}}$, were considered too low, and were not taken into account when computing the geometric stiffness matrix. GBT analyses were carried out considering different deformation modes and number of elements per part, and the results concerning $\sigma_{\mathrm{x}}$ were compared. In Figs. 13 and 14, it can be observed the sensitivity of the stress distribution to the modes included in the analysis. The use of only conventional modes (D,G and L) does not lead to accurate distributions (Figs. 13(b) and 13(c), and GBT DG and GBT DG+L in Fig. 14). The key point is the participation of the local shear modes (LS). When such modes are considered (Figs. 13(d) to 13(g), and options with LS modes in Fig. 14), the stresses become similar to those obtained with SFEM. 
The use of the natural shear (NS) and transverse extension (TE) modes does not involve a significant improvement of the longitudinal stress distribution (Fig. 14). The effect of natural shear modes is always very small (see also next Sections), and the effect of the transverse extension modes has been verified to be only significant in the determination of $\sigma_{s}$ distribution.

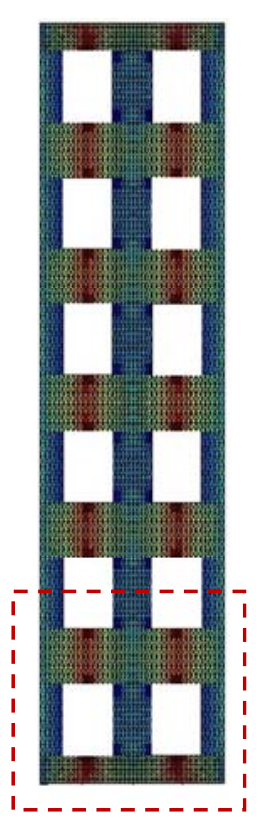

a)

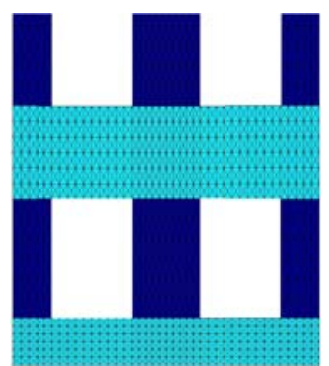

b) DG

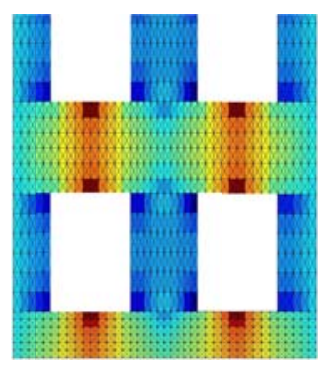

e) $\mathrm{DG}+\mathrm{L}+\mathrm{LS}$

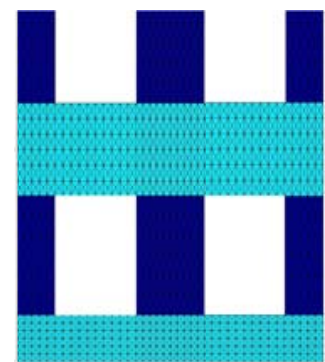

c) $\mathrm{DG}+\mathrm{L}$

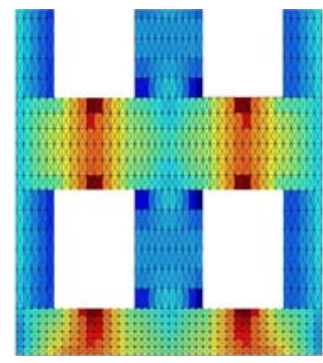

f) $D G+L+L S+T E$

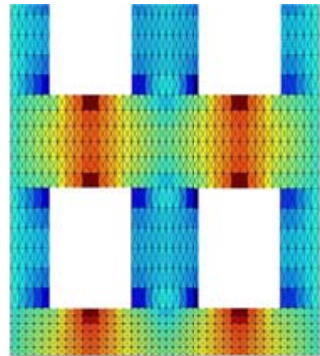

d) DG+LS

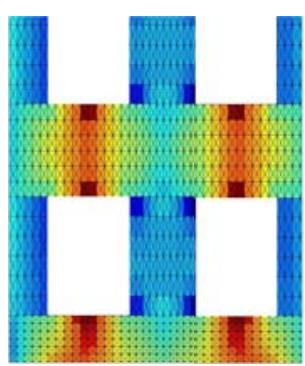

g) $\mathrm{DG}+\mathrm{L}+\mathrm{LS}+\mathrm{TE}+\mathrm{NS}$

Fig. 13. Sensitivity of $\sigma_{\mathrm{x}}$ to the deformation modes included in the analysis (the map of stresses corresponds to the sheet outer plane).

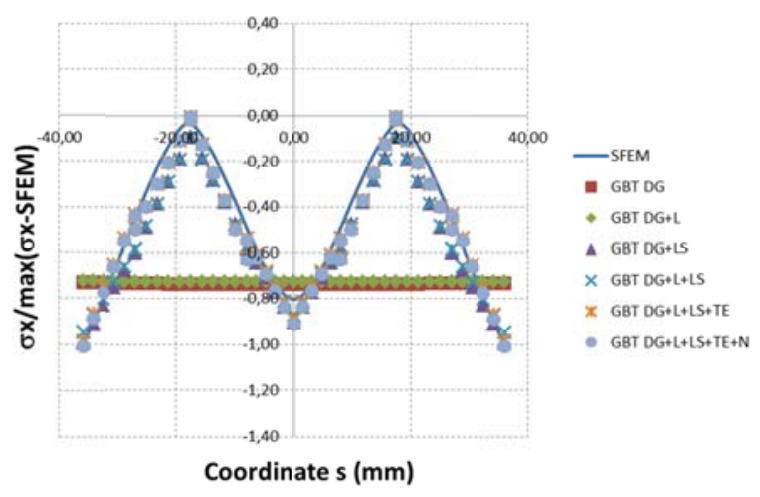

a) Section $\mathrm{A}$

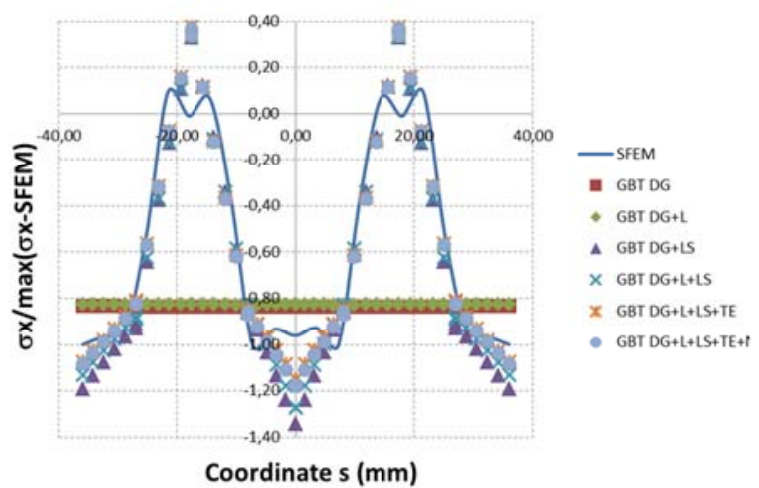

b) Section B

Fig. 14. Sensitivity of $\sigma_{\mathrm{x}}$ in sections A and B (Fig. 11) to the deformation modes included in the analysis. 
The sensitivity of the $\sigma_{x}$ stresses to the number of finite element divisions can be observed in Fig. 15. The number of cross-section nodes is 15 for all the analyses (see discussions in Section 3.5), while the number of elements in the longitudinal direction goes from 1 to 6 elements per part. The $\sigma_{\mathrm{x}}$ gradient in the longitudinal and transversal directions can be roughly captured with only one finite element. The GBT stress distribution obviously improves as the number of finite elements increases. Good results are already obtained when four elements per part are used (Fig. 15(c)).

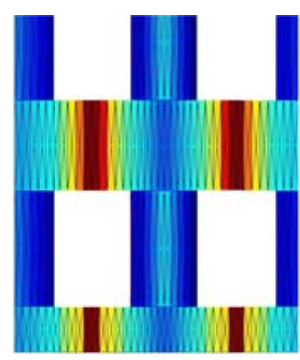

a) 1 element

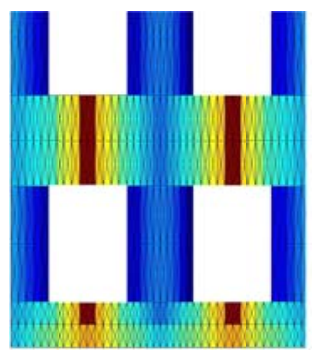

b) 2 elements

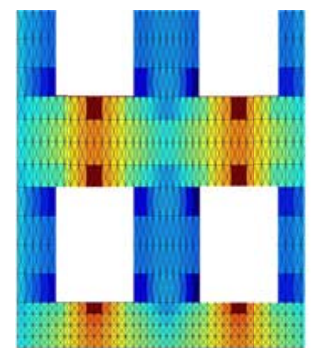

c) 4 elements

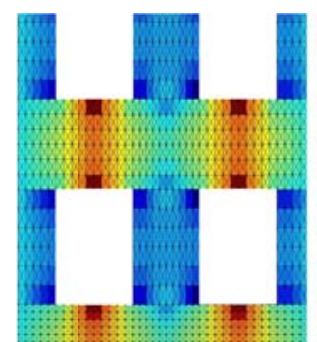

d) 6 elements

Fig. 15. Sensitivity of $\sigma_{\mathrm{x}}$ to the number of GBT beam elements per part (the map of stresses corresponds to the sheet outer plane).

Finally, it is worth noting that when pure compression is applied, the stress resultants and stress distributions are the same all along the member for each one of the parts, perforated and non-perforated parts (see stresses in Fig. 13(a)). It is recommended to take advantage of this fact, because it significantly reduces the computational cost when constructing the global geometric stiffness matrix. For instance, in the present investigation, only four different geometric stiffness submatrices were used: (i) intermediate perforated part; (ii) intermediate non-perforated part; (iii) top non-perforated part; and (iv) bottom non-perforated part.

\subsection{Selection of deformation modes}

\subsubsection{Distortional and global buckling loads}

Once a robust procedure had been set by selecting the appropriate constraint equations and connecting points, and the relevant initial stresses had been identified, the investigation focused on the selection of the deformation modes to be included in the buckling analysis.

Before going into details, it should be pointed out that all combinations of deformation modes tested in the investigation contained all distortional and global conventional modes (the DG combination): the axial extension mode, two flexural modes, the torsional mode and all distortional modes. Some preliminary analyses carried out on column S4 without these modes did not succeed (no solution could be obtained). Consequently, it was considered that the DG combination should always be included in the analyses to ensure the correct performance of the procedure. The actual goal of this part of the article is to show which modes have to be added to the DG combination to get accurate buckling load estimations.

In the beginning, the procedure was developed to calculate distortional and global buckling loads, since it was not expected to obtain good results for local buckling with the proposed GBT procedure (see Casafont et al. ${ }^{22}$ ). This is the reason why the article shows the results for distortional and global buckling modes first, and afterwards, in the next Section, local buckling is investigated. Furthermore, it will be seen that the optimum selection of deformation modes for local buckling is not optimum for the other buckling modes.

The first analyses of S4 that produced reasonable results were carried out considering the mentioned DG combination. The accuracy of the analyses is shown in Figs. 16(a) and 16(b). 
It can be observed a first range of column lengths, until approximately $1500 \mathrm{~mm}$, where the $\mathrm{P}_{\mathrm{b}}$ ${ }_{\mathrm{GBT}} / \mathrm{P}_{\mathrm{b}}$ SFEM ratios are not stable. They decrease for distortional buckling and increase for torsional-flexural buckling. This is attributed to the fact that in this first range, the symmetric distortional mode is combined with local modes, and the torsional-flexural mode is combined with anti-symmetric distortional modes. As the length increases, the participation of these secondary modes disappears and the results become more stable.

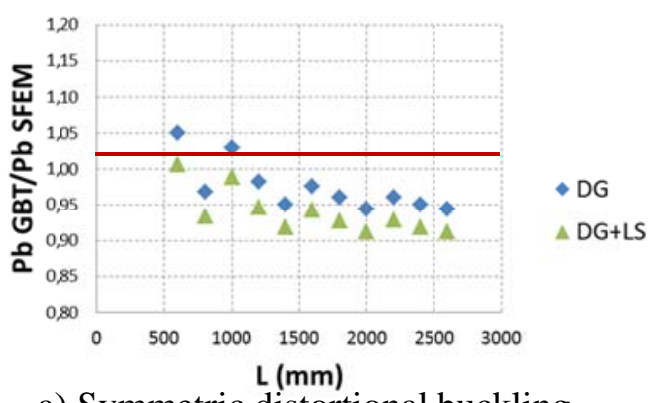

a) Symmetric distortional buckling

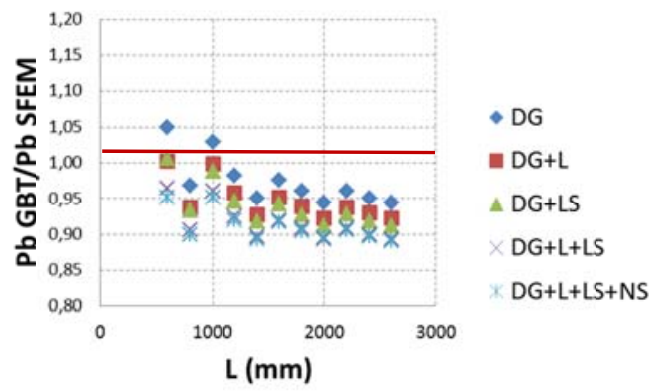

c) Symmetric distortional buckling

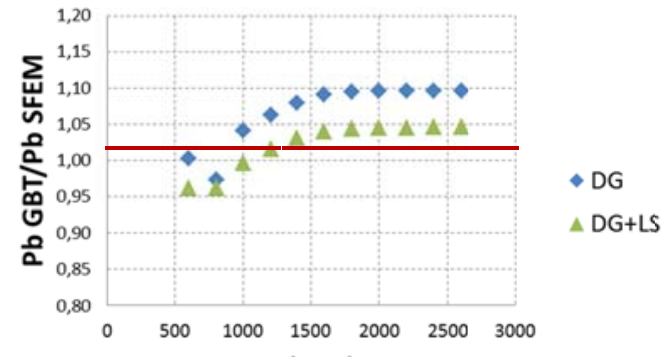

b) Torsional-ftexural buckling

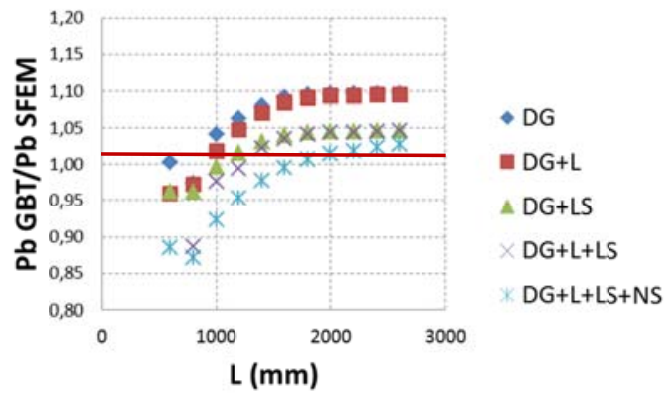

d) Torsional-flexural buckling

Fig. 16. Accuracy of the first analyses on S4.

When looking at the results above $1500 \mathrm{~mm}$, it can be observed that the proposed GBT analysis produces slightly conservative distortional buckling loads, but global buckling loads are overestimated. The $\mathrm{P}_{\mathrm{b} \text { GBT }} / \mathrm{P}_{\mathrm{b}}$ SFEM ratios go up to 1.10 for global bucklling (or even higher in some of the other columns investigated).

In order to improve the results, local shear modes were added to the analysis (DG+LS combination). This was the first option in view of the results of the stress study presented in the previous Section, where it was verified that the inclusion of local shear modes was fundamental to reproduce the distribution of the longitudinal normal stresses $\left(\sigma_{\mathrm{x}}\right)$. The new DG+LS buckling loads, shown also in Figs. 16(a) and 16(b), are lower, becoming acceptable for the TF buckling mode. TF $\mathrm{P}_{\mathrm{b} \text { GBT }} / \mathrm{P}_{\mathrm{b} \text { SFEM }}$ ratios decrease to about 1.05.

Furthermore, it is also interesting to see Fig. 17, that shows the normalized longitudinal displacement distribution in the web of a non-perforated and perforated column. The plot corresponds to cross-section B highlighted in Fig. 11(a), and to a dominant torsional-flexural buckling mode. The displacements of the graph have been normalized with respect to the maximum web displacement. It can be observed that the non-perforated column shows a linear displacement distribution, while for the perforated one the distribution becomes slightly non-linear. DG deformation modes can only produce linear longitudinal displacements. Therefore, it seems reasonable to add the LS modes to improve the results of the DG combination. It is a way to allow the GBT model to adapt to the non-linear distribution (see Fig. 2(c)). 


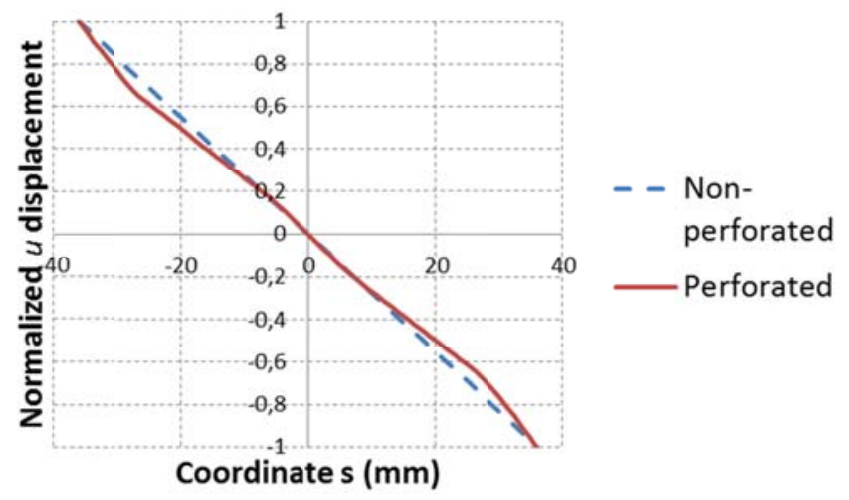

Fig. 17. SFEM distribution of longitudinal web displacements for a TF buckling mode. Values normalized with respect to the maximum web longitudinal displacement.

New mode combinations are studied to provide a complete view of the sensitivity of the results with respect to the deformation modes. The deformation mode sets finally investigated are the DG and DG+LS discussed in the previous paragraphs, together with: $\mathrm{DG}+\mathrm{L}$ (distortional + global + conventional local modes), DG $+\mathrm{L}+\mathrm{LS}$ (distortional + global + conventional local + local shear modes), and DG+L+LS+NS (distortional + conventional local + local shear + natural shear modes). Transverse extension modes are not considered in the study of distortional and global buckling loads, because they involve a huge computational cost (as it will be seen in the next Section).

Figs. 16(c) and 16(d) show the results obtained with the different sets of deformation modes. On the one hand, it can be observed that distortional buckling loads become more conservative as the number of deformation modes are added to the analyses. On the other hand, concerning the torsional flexural buckling mode, it is interesting to see that: (i) the DG+L combination does not improve the DG set, because LS deformation modes are not used; and (ii) the accuracy of the procedure improves with the number of deformation modes.

In Fig. 16, it seems difficult to choose a mode combination from the accuracy point of view. However, the decision becomes easy when the computational cost is considered. This can be seen in Fig. 18(a), that shows the CPU time cost of each option normalized to the time cost of the simplest mode combination, the DG combination. It is noted that 90 seconds were needed to produce the whole set of DG buckling loads shown in Fig. 9(e) (or Fig. 16) with a non-optimized Matlab code in a PC Intel Core i5-3470 CPU3.2Ghz. From the values shown in the figure, it is reasonable to state that the DG+LS option guarantees a good balance between accuracy and computational cost.

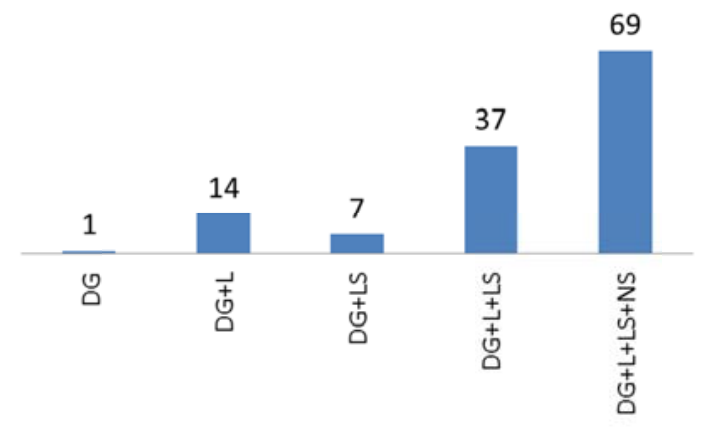

a) Distortional and global buckling

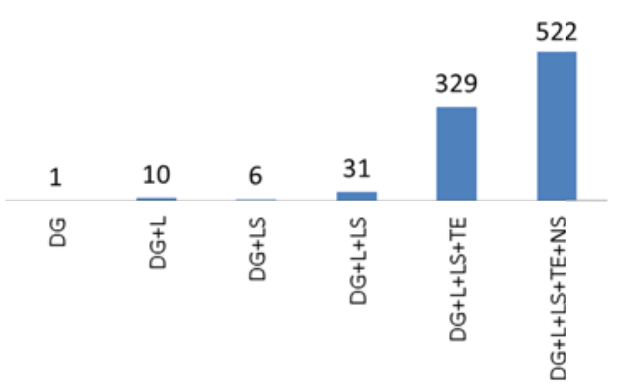

b) Local buckling

Fig. 18. Normalized CPU time cost. 
The DG+LS combination was finally chosen to assess the performance of the proposed procedure. Fig. 19(a) shows the accuracy resulting from the analyses of all the columns presented in Section 3.1 (Figs. 5 and 6). It can be concluded that rather good buckling load

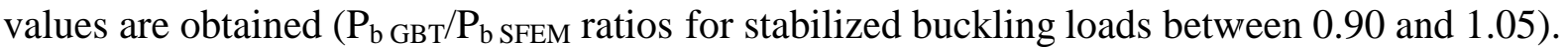

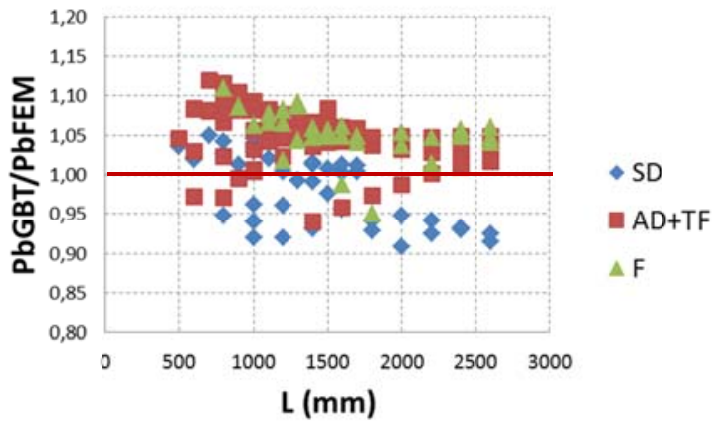

a) Distortional and global buckling

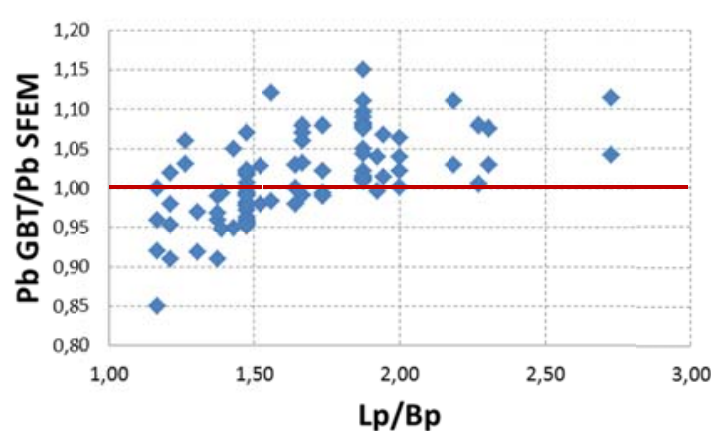

b) Local buckling

Fig. 19. Accuracy of the proposed method when tested on columns shown in Figs. 5 and 6.

\subsubsection{Local buckling loads}

In a similar way as for the distortional and global buckling loads, different mode sets were investigated with the aim to obtain the optimum combination for local buckling. The tested sets were: $\mathrm{DG}+\mathrm{L}, \mathrm{DG}+\mathrm{L}+\mathrm{LS}, \mathrm{DG}+\mathrm{L}+\mathrm{LS}+\mathrm{TE}$ (distortional + global + local shear + transverse extension modes), and DG+L+LS+TE+NS (distortional + global + local shear + transverse extension + natural shear modes).

The resulting local buckling loads for cross-section S4 are shown in Fig. 20(a). It can be seen that rather good results are already obtained using the first and simplest mode combination (DG $+\mathrm{L})$, with $\mathrm{P}_{\mathrm{b}} \mathrm{GBT}_{\mathrm{P}} / \mathrm{P}_{\mathrm{b}}$ SFEM ratios between 1.00 and 1.05. Actually, when LS modes are added, buckling loads become more unconservative, and they only can be considered acceptable when TE and NS modes are also included in the analysis. The problem with such sets combining so many deformation modes is again the computational cost, as can be seen in Fig. 18(b). The CPU time was so high that it was believed impossible to analyse all columns presented in Section 3.1 to assess the performance of the method. It should be noted that, for instance, the computational time of the DG+L+SL+TE set is more than 30 times higher the computational time of the DG+L combination. In a similar way as in the previous section, the computational cost in Fig. 18(b) has been normalized to the computational cost of the DG option, which was 20 seconds (the results of the DG option are not included in Fig. 20(a)).

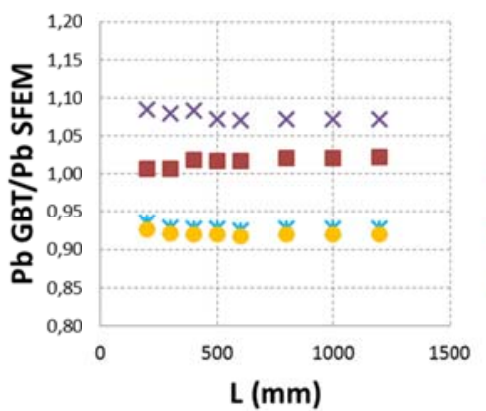

a) Local buckling

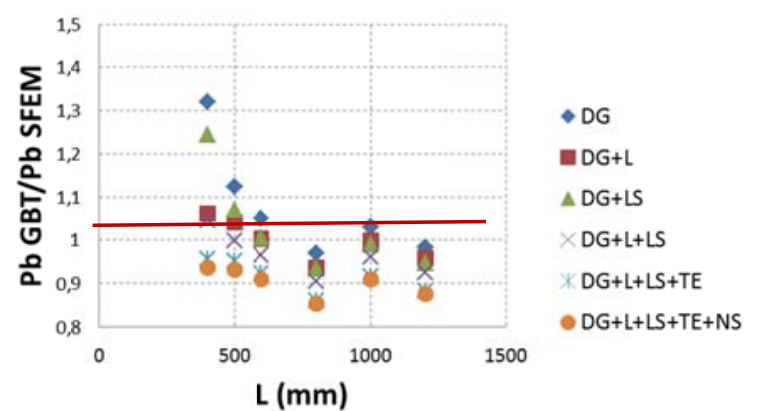

b) Distortional buckling;

Fig. 20. Accuracy of the proposed method when tested on short collumns. 
Finally, the DG+L option was chosen to assess the local buckling load predictions. The results are better than expected, with most of the $\mathrm{P}_{\mathrm{b} \text { GBT }} / \mathrm{P}_{\mathrm{b}}$ SFEM ratios between 0.90 and 1.10 (See Fig. 19(b)).

It is also worth to point out that the GD+L combination may be used to improve the distortional buckling loads of short columns, which usually show distortional buckling modes that are combined with local buckling modes. This can be observed in Fig. 20(b), where distortional buckling loads obtained with the DG+LS set are compared to those obtained $\mathrm{DG}+\mathrm{L}$ set (and others) for S4 short columns.

\subsection{Sensitivity to the cross-section and member discretization}

The sensitivity of the procedure to the model discretization is first illustrated with examples working on channel columns (column S4). Afterwards, at the end of the Section, advice will be given concerning the optimum discretization for models of omega columns.

All calculations on channel columns performed in the preceding Sections were carried out with the cross-section shown in Fig. 8(a), where 15 nodes are used. Concerning the member (or longitudinal) discretization, the number of finite elements was different depending on the buckling mode type calculated: 1 element was used for distortional and global buckling loads, and 2 elements were used for local buckling loads.

\subsubsection{Sensitivity to the number of cross-section segments}

First, the sensitivity to the number of cross-section nodes is investigated. It should be pointed out that reducing the number of cross-section nodes will reduce the number of deformation modes considered in the analysis of the member and, therefore, the degrees of freedom and the computational cost. The number of modes considered in a GBT beam analysis is actually independent of the cross-section discretization, because it is always possible to include just some of the modes that result from the GBT cross-section analysis. However, in the proposed procedure all modes derived in the cross-section analysis for a specific family are included in the calculation of the buckling load. For instance, if it is decided to consider the LS modes, then all LS modes are included in the member analysis. This is done in this way to avoid having to select different connecting point configurations depending on the number and shape of deformation modes chosen for the analysis. It is a way of reducing the number of decisions to be made during the procedure.

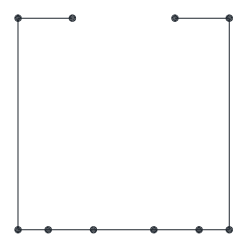

a) 10 nodes

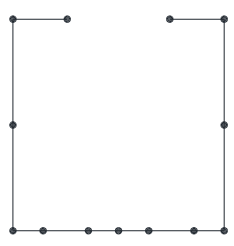

b) 13 nodes

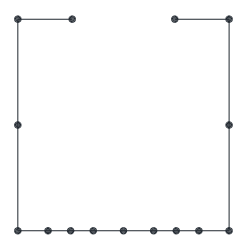

c) 15 nodes

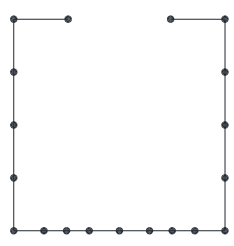

d) 19 nodes

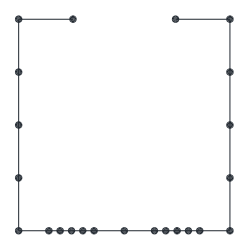

e) 23 nodes

Fig. 21. Cross-section discretization investigated for column S4.

Fig. 22 shows the local, distortional and global buckling loads obtained for four different S4 column lengths analysed with the cross-section models shown in Fig. 21. The DG+L mode set was used for the local buckling mode, and the DG+LS mode set for distortional and global buckling loads, according to the conclusions drawn in the previous Section. Concerning local buckling loads, 15, or even 13, nodes are enough to get accurate values; while for distortional and global buckling loads, it can be observed that acceptable results are already obtained with 
only 10 nodes, which is the number of nodes needed to define a channel cross-section with two perforations.

Finally, it is decided that 15 nodes is a good option for the buckling load calculation of channel rack cross-sections, such as S4.

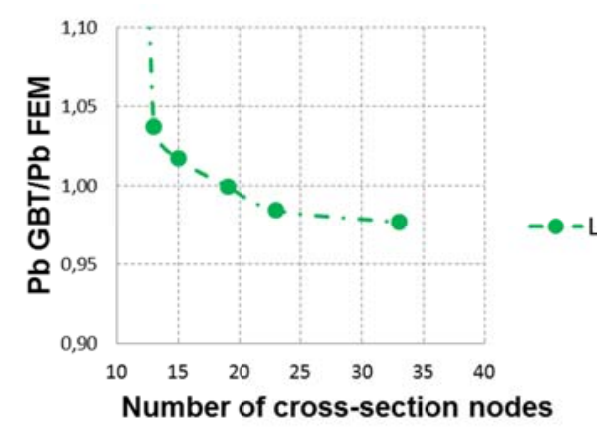

a) $\mathrm{L}=500 \mathrm{~mm}$

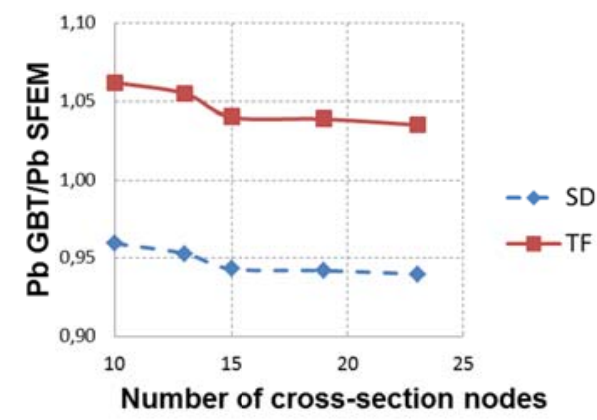

c) $\mathrm{L}=1600 \mathrm{~mm}$

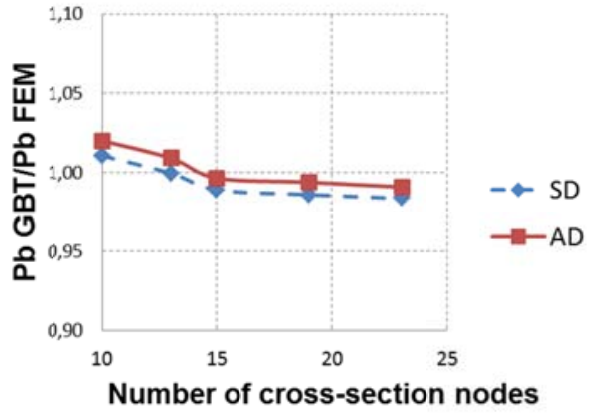

b) $\mathrm{L}=1000 \mathrm{~mm}$

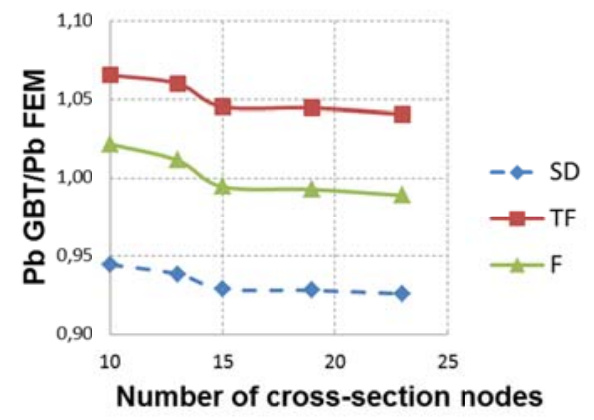

d) $\mathrm{L}=2200 \mathrm{~mm}$

Fig. 22. Sensitivity to the number of cross-section nodes (L: local buckling loads; SD: symmetric distortional buckling loads; TF: torsional-flexural buckling loads; F: flexural buckling loads).

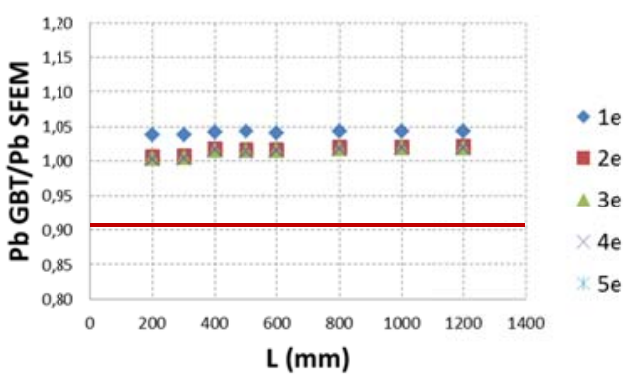

a) Local buckling loads

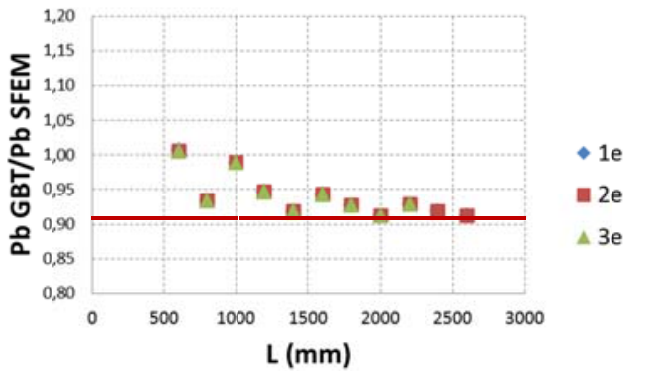

b) Symmetric distortional buckling loads.

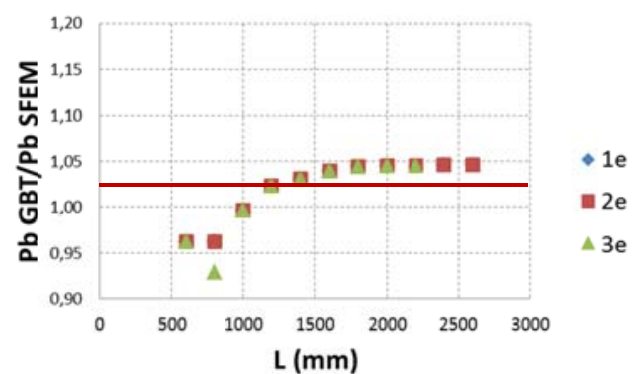

c) Torsional-flexural buckling loads.

Fig. 23. Sensitivity to the member discretization (for example: 1e means one element per part). 


\subsubsection{Sensitivity to the number of finite elements}

The longitudinal discretization is high in rack columns, because their pitch (Figs. 4 and 5) is short compared to their length. Many elements have to be used to reproiduce the perforated geometry. As a consequence, the simplest possible longitudinal discretization, one finite element per part, is enough to get accurate results of distortional and global buckling loads, see Fig. 23(b) and 23(c). This is due to the fact that the distortional and global buckling wavelengths are much longer than the pitch of the columns.

On the contrary, if the calculation is carried out to determine local buckling loads, it is recommended to perform always a sensitivity analysis of the results to the number of longitudinal beam elements. The buckling wavelengths of local modes may be similar or shorter than the pitch lengths in rack columns. Consequently, the buckling load becomes sensitive to the longitudinal discretization, and more than one element per part is needed. For example, 2 beam elements have to be considered in the analysis of the S4 column to make the model insensitive to the discretization, as demonstrated in Fig. 23(a).

The conclusion in relation to the longitudinal discretization of S4 can be that using 2 elements per part will ensure accurate results for all types of buckling modes. If local buckling loads are not needed, than it is recommended to use just 1 element per part.

It should be pointed out that when more than 1 element is used in the perforated parts, and local deformation modes are considered, the eigen-buckling analysis results in spurious buckling modes localized at the perforated zones of the pitch, as can be seen in Fig. 24. Such buckling modes usually show a very low buckling load, because the stiffness of the perforated area, with reduced thickness, is small. There is no need to worry about these spurious modes because they are very easy to identify and remove from the output of the analysis.

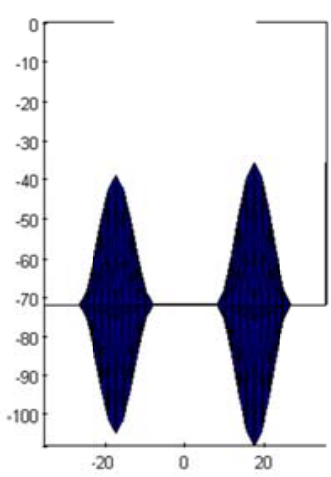

Fig. 24. Spurious local buckling mode.

Finally, it is noted that Table 1 is showing the optimum section discretization for the omega rack columns analysed in the present investigation. Concerning the longitudinal discretization, 3 elements per part were needed in such columns to get accurate local buckling loads, while only 1 element was enough for the distortional and global buckling loads.

\section{CONCLUSIONS}

The first results of an investigation on the analysis of cold-formed steel rack columns with multiple perforations by means of the Generalised Beam Theory are presented. From the beginning, the aim of the authors was to develop a general method with a wide range of application, where perforations had to be considered in the derivation of the analysis 
procedure. The idea was to avoid the tough task of calibrating the method, as it occurs in the approaches based on the use of reduced thicknesses.

The problem of dealing with perforations has been solved in GBT by meshing the perforated and non-perforated parts of the member with two different finite elements, the perforated and non-perforated finite element. Afterwards, constraint equations are used to link the degrees of freedom at the interface cross-section between the parts. The main difficulties encountered during the development of the procedure have been: (i) the robustness of the connection at the interface; and (ii) the accuracy of results, especially, for global buckling.

The robustness of the procedure has been achieved by selecting the proper constraint equations and connecting points for each family of GBT deformation modes. Different constraint options were tested in the investigation to finally propose those that result in coherent buckling loads and buckling modes. Furthermore, the constraint equations have been set so that no decision has to be made by the user of the method concerning which deformation modes (within each family) and connection points should be considered in the analysis. This was also one of the objectives of the investigation.

On the other hand, the article also recommends which families of deformation modes have to be used to get accurate results. In these sense, it is very important the role of the local shear modes when calculating global buckling loads. The accuracy finally achieved is considered acceptable, although, for instance, distortional buckling loads are slightly underestimated, and global buckling loads tend to be overestimated.

Future work will be focused on different issues: (i) to improve de current degree of accuracy; (ii) to optimise the computation procedure so that the analysis can be performed in an effective way with as many deformation modes as possible; (iii) additional validation of the procedure by testing more complex members and load conditions; and, finally, (iv) to investigate the effect of considering additional stress resultants when constructing the geometric stiffness matrix.

\section{REFERENCES}

1. Z. Li and B.W. Schafer, Buckling analysis of cold-formed steel members with general boundary conditions using CUFSM: conventional and constrained finite strip methods, Proceedings of the $20^{\text {th }}$ International Specialty Conference on Cold-Formed Steel Structures (St. Louis, MO, 2010).

2. GBTUL Version 2.0.4.0, The Generalised Beam Theory Research Group, Instituto Superior Tecnico, Lisbon (2013).

3. D. Camotim, C. Basaglia, R. Bebiano, R. Gonçalves and N. Silvestre, Latest developments in the GBT analysis of thin-walled steel structures, Proc. Int. Coll. Stability and Ductility of Steel Struct. (Rio de Janeiro, Brazil, 2010).

4. C. Basaglia, D. Camotim, Buckling Analysis of Thin-Walled Steel Structural Systems Using Generalized Beam Theory (GBT), International Journal of Structural Stability and Dynamics, 15(1) (2015).

5. M. Casafont, M.M. Pastor, J. Bonada, F. Roure and T. Peköz, Linear buckling analysis of perforated steel storage rack columns with the Finite Strip Method, Thin-Walled Structures, 61 (2012) 71-85.

6. C.D. Moen and B.W. Schafer, Elastic buckling of cold-formed steel columns and beams with holes, Engineering Structures, 31 (2009) 2812-2824.

7. F.H. Smith and C.D. Moen, Finite strip elastic buckling solutions for thin-walled metal columns with perforation patterns, Thin-Walled Structures, 79 (2014) 187-201.

8. G. Eccher, K.J.R. Rasmussen and R. Zandonini, Elastic buckling analysis of perforated thinwalled structures by the isoparametric spline finite strip method, Thin-Walled Structures, 46 (2008) 165-191. 
9. M. Casafont, F. Marimon, M.M. Pastor and M. Ferrer, Linear buckling analysis of thin-walled members combining the Generalised Beam Theory and the Finite Element Method, Computers and Structures, 89(21-22) (2011) 1982-2000.

10. J. Bonada , M. Casafont, F. Roure and M.M. Pastor, Selection of the initial geometric imperfection in nonlinear of cold-formed steel rack columns, Thin-walled structures, 51 (2012) 91-111.

11. J.Cai and C.D. Moen, Elastic buckling analysis of thin-walled structural members with rectangular holes using generalised theory, Thin-Walled Structures, 98 (2016) 518-532.

12. R. Schard, Verallgemeinerte technische biegetheorie (Springer Verlag, Heidelberg, 1989).

13. N. Silvestre, D. Camotim, First-order generalised beam theory for arbitrary orthotropic materials, Thin-walled structures, 40(9) (2002) 791-820.

14. R. Gonçalves, M. Ritto Corrêa and D. Camotim, A new approach to the calculation of crosssection deformation modes in the framework of generalised beam theory, Computational Mechanics, 46 (2010) 759-781.

15. R. Bebiano , N. Silvestre and D. Camotim, GBT formulation to analyse the buckling behavior of thin-walled members subjected to non-uniform bending, International Journal of Structural Stability and Dynamics, 7(1) (2007) 23-54.

16. C. Basaglia, D. Camotim and N. Silvestre, Post-buckling analysis of thin-walled steel frames using generalised beam theory (GBT), Thin-walled structures, 62 (2013) 229-242.

17. M. Casafont, J. Bonada, M.M. Pastor and F. Roure, GBT Calculation of elastic buckling loads of cold-formed steel rack columns, Proceedings of the International Colloquium on Stability and Ductility of Steel Structures-SDSS 2016 (Erns \& Sohn, Portugal, 2016).

18. MATLAB and Statistics Toolbox Release 2012, The MathWorks, Inc., Natick, Massachusetts, United States.

19. ANSYS $^{\mathrm{R}}$ Academic, release 14.0. SAS IP, Inc (2011).

20. C. Basaglia, D. Camotim and N. Silvestre, Global buckling analysis of plane and space thin-walled frames in the context of GBT, Thin-walled structures, 46 (2008) 79-101.

21. C. Basaglia, D. Camotim and N. Silvestre, GBT-based local, distortional and global buckling analysis of thin-walled steel frames, Thin-walled structures, 47 (2009) 1246-1264.

22. M. Casafont, J. Bonada, M.M. Pastor and F. Roure, GBT Calculation of distortional and global buckling loads of cold-formed steel channel columns with multiple perforations, Eighth International Conference on Advances in Steel Structures (Lisbon, Portugal, 2015). 